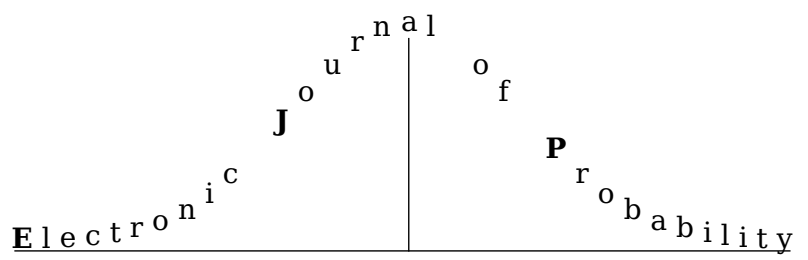

Electron. J. Probab. 26 (2021), article no. 14, 1-33.

ISSN: 1083-6489 https://doi.org/10.1214/21-EJP594

\title{
Spectral theory for one-dimensional (non-symmetric) stable processes killed upon hitting the origin*
}

\author{
Jacek Mucha ${ }^{\dagger}$
}

\begin{abstract}
We obtain an integral formula for the distribution of the first hitting time of the origin for one-dimensional $\alpha$-stable processes $X_{t}$, where $\alpha \in(1,2)$. We also find a spectraltype integral formula for the transition operators $P_{t}^{\mathbb{R} \backslash\{0\}}$ of $X_{t}$ killed upon hitting the origin. Both expressions involve exponentially growing oscillating functions, which play a role of generalised eigenfunctions for $P_{t}^{\mathbb{R} \backslash\{0\}}$.
\end{abstract}

Keywords: stable process; spectral theory; hitting time; transition density. MSC2020 subject classifications: Primary 60G51; 60G52, Secondary 60J35; $60 \mathrm{~J} 45$. Submitted to EJP on October 28, 2019, final version accepted on January 30, 2021. Supersedes arXiv: 1910.12821.

\section{Introduction}

The main purpose of this article is to extend the results of [23], where a large class of symmetric Lévy processes was considered, to non-symmetric stable Lévy processes. For such a process $X_{t}$, we study the hitting time of the origin, and transition operators of the process $X_{t}$ killed upon hitting the origin. We construct appropriate 'generalised eigenfunctions' $F^{+}(s x)$ and $F^{-}(s x)$, and we provide expressions similar to those of [23]. In our case, however, the functions $F^{+}$and $F^{-}$are no longer bounded; in fact, they grow exponentially fast, and thus the methods of [23] need to be substantially modified. Our approach is similar to that of [21], where similar problems for hitting a half-line are studied. However, we avoid the use of special functions. Instead, we consider integraltype expressions, related to some extend to [25]; see also the prelimiary version [24] of that article.

Hitting times for Markov processes are one of the fundamental objects in probabilistic potential theory, with numerous applications to other areas of mathematics. Various results for the Brownian motion are collected in [5], Appendix 1. Hitting times for

\footnotetext{
${ }^{*}$ Work supported by National Science Centre (NCN), Poland, under grant 2015/19/B/ST1/01457

${ }^{\dagger}$ Faculty of Pure and Applied Mathematics, Wrocław University of Science and Technology ul. Wybrzeże Wyspiańskiego 27 50-370 Wrocław, Poland.

E-mail: jacek.mucha@pwr.edu.pl
} 
symmetric stable Lévy processes have already been studied in 1960s, see, for example, [3]. In [10] a formula for the density of the hitting time of the origin was obtained for spectrally positive Lévy processes. The theory was further developed in [32], and in [34] a series expansion of the density of the hitting time of the origin was found for stable processes with no negative jumps. Further results for completely asymmetric stable processes were presented in [43], where series representations for the density are presented, and in Section 46 of [41]. The Mellin transform for the hitting time of zero is given in [20]. Additionally, in [43], hitting times of points are proved to be unimodal when $\alpha \leqslant 3 / 2$. Later, in [28], unimodality was proven for $\alpha \in(1,2]$. More general results about unimodality of hitting times for Markov processes can be found in [40]. Asymptotic analysis of the hitting times of points can also be found in [17, 35, 46]. Estimates for hitting times of points for more general symmetric Lévy processes were obtained under some mild regularity assumptions in [14].

Obviously, hitting times and distributions for stable processes have been studied also for more general sets. These results are, however, of much different nature, and we only mention some examples that are at least remotely related to our work. Hitting distributions of the interval $[-1,1]$ or its complement $\mathbb{R} \backslash(-1,1)$ have been found in [27] and [38], respectively; see also [36] for further discussion and references. Hitting times of half-lines, called first passage times, are of particular interest, being the main subject of fluctuation theory for Lévy processes; we mention here [13, 19, 21].

As mentioned above, spectral theory of symmetric Lévy processes killed upon hitting $\{0\}$ is developed in [23]. Further work in this area can be found in [16], where a narrower class of symmetric Lévy processes with completely monotone jumps is studied. Similar work for symmetric processes in half-line can be found in [22] and [26], which extend the former work [18] on the Cauchy process. Non-symmetric stable processes in half-line have been studied in a similar way in [21]; see also [24, 25] for preliminary results for more general non-symmetric Lévy processes with completely monotone jumps. We note that spectral theory of non-symmetric Markov processes on the half-line was also studied in [29] (one-dimensional diffusion processes), [31] (branching processes) and [33] (non-self-adjoint Markov semigroups).

Our main goal is to extend the results from [23] to the class of non-symmetric $\alpha$-stable processes, $\alpha \in(1,2)$. The symmetric case is much easier, mainly because in this case the characteristic exponent of the process is real-valued. This property is crucial for the method developed in [23]. However, the tools developed in [21] allow us to follow some of the arguments from [23], after appropriate deformation of the contour of integration to the line along which characteristic exponent takes real values.

Let us briefly motivate the form of our main result, Theorem 1.1. If $\tau$ is the hitting time of the complement of a compact set $D$ for a sufficiently regular symmetric Markov process $X_{t}$, then the transition operators $P_{t}^{D}$ of the process $X_{t}$ killed at $\tau$ are compact operators on $L^{2}(D)$, and it is easy to find spectral expansion of $\mathbb{P}^{x}(\tau>t)$ : we have

$$
\mathbb{P}^{x}(\tau>t)=\sum_{n=1}^{\infty} e^{-\lambda_{n} t} \varphi_{n}(x) \int_{D} \varphi_{n}(y) d y,
$$

where $\left(\varphi_{n}: n=1,2, \ldots\right)$ is a complete orthonormal system of eigenfunctions of $P_{t}^{D}$, with corresponding eigenvalues $e^{-\lambda_{n} t}$. We refer to [12] for a rigorous discussion of an analogous description of the transition density (the kernel of $P_{t}^{D}$ ). When $D$ is unbounded and $P_{t}^{D}$ fail to be compact operators, one can expect that a continuous variant of the above expansion holds:

$$
\mathbb{P}^{x}(\tau>t)=\int_{S} e^{-\lambda(s) t} \varphi_{s}(x)\left(\int_{D} \varphi_{s}(y) d y\right) m(d s),
$$


where $\varphi_{s}$ are generalised eigenfunctions (or resonanses) of $P_{t}^{D}$ with generalised eigenvalues $\lambda(s)$. Here $S$ is some parameter set, and $m$ is an appropriate meusure on $S$; again we refer to [12] for a rigorous discussion of such expansion for transition densities. Similar problem for non-symmetric processes are generally much harder. However, in certain cases one can hope for similar expansions. In the compact case, if $P_{t}^{D}$ admit a complete system of eigenfunctions $\varphi_{n}^{-}$and co-eigenfunctions $\varphi_{n}^{+}$, then it is expected that

$$
\mathbb{P}^{x}(\tau>t)=\sum_{n=1}^{\infty} e^{-\lambda_{n} t} \varphi_{n}^{-}(x) \int_{D} \varphi_{n}^{+}(y) d y .
$$

Similar expressions are possible in the non-compact case, of the form

$$
\mathbb{P}^{x}(\tau>t)=\int_{S} e^{-\lambda(s) t} \varphi_{s}^{-}(x)\left(\int_{D} \varphi_{s}^{+}(y) d y\right) m(d s) .
$$

The article [21] derives a formula of the form given above for the first exit time from $(0, \infty)$ for non-symmetric $\alpha$-stable processes. Here we prove an analogous result for the hitting time of 0 . In either case the generalised eigenfunctions $\varphi_{s}^{-}(x)=F(s x)$ have exponential growth; namely, we (roughly) have $F(x)=e^{a x} \sin (b x+c)-G(x)$ for a reasonably small remainder term $G$. As we shall see below, this rapid growth of $F$ is a constant source of problems in applications of Fubini's theorem, invertions of Laplace transforms etc.

Much of the inspiration for the present work also came from the theory of Rogers functions $([24,25])$. The characteristic exponent of a stable process is a particularly simple example of a Rogers function. Many of the results presented below seem to extend to more general Rogers functions, which suggests that our main results can possibly be extended to more general Lévy processes with completely monotone jumps.

Distribution of hitting times of the points, as mentioned above, is widely studied and useful in many applications. For example, in [20] it is used (in different form) to study stable processes conditioned to avoid zero. Such objects appear also in the proofs in excursion theory $([7,20])$, potential theory $([4])$ and local times (Second Ray-Knight theorem, [30]), not to mention financial mathematics ([47]). Our formula may help with describing the asymptotics of hitting times (as in [16]). Our results concerning generalised eigenfunctions, on the other hand, may provide even more interesting applications. Such objects invariant under operator of rather standard form seem to be commonly desired.

\subsection{Main results}

Let $X_{t}$ be the $\alpha$-stable process with index $\alpha \in(1,2)$ and positivity parameter $\rho \in$ $\left[1-\frac{1}{\alpha}, \frac{1}{\alpha}\right]$. We assume that $X_{t}$ is normalised in such a way that if $\psi$ is the characteristic exponent of $X_{t}$, then $|\psi(1)|=1$. Let

$$
\theta=(1-2 \rho) \frac{\pi}{2},
$$

so that $\psi(\xi)=e^{-i \alpha \theta}|\xi|^{\alpha}$ for $\xi>0$ and $\psi(\xi)=e^{i \alpha \theta}|\xi|^{\alpha}$ for $\xi<0$. We remark that our assumption $\alpha \in(1,2)$ is not restrictive: if $\alpha \leqslant 1$, then, with probability one, $X_{t}$ never hits 0 .

Define the functions $G^{+}$and $G^{-}$by the formulas

$$
\begin{aligned}
& G^{+}(x)=G^{-}(-x)=\frac{\alpha \sin \frac{\pi}{\alpha}}{\pi} \int_{0}^{\infty} \frac{t^{\alpha} \sin \left(\alpha\left(\frac{\pi}{2}-\theta\right)\right)}{t^{2 \alpha}-2 t^{\alpha} \cos \left(\alpha\left(\frac{\pi}{2}-\theta\right)\right)+1} e^{-t x} d t \\
& G^{+}(-x)=G^{-}(x)=\frac{\alpha \sin \frac{\pi}{\alpha}}{\pi} \int_{0}^{\infty} \frac{t^{\alpha} \sin \left(\alpha\left(\frac{\pi}{2}+\theta\right)\right)}{t^{2 \alpha}-2 t^{\alpha} \cos \left(\alpha\left(\frac{\pi}{2}+\theta\right)\right)+1} e^{-t x} d t
\end{aligned}
$$


for $x>0$. Define furthermore

$$
F^{+}(x)=F^{-}(-x)=e^{-x \sin \theta} \sin \left(|x| \cos \theta+\theta \operatorname{sign} x+\frac{\pi}{\alpha}-\frac{\pi}{2}\right)-G^{+}(x)
$$

for $x \in \mathbb{R} \backslash\{0\}$ with $F^{+}(0)=0$. Note that $G^{-}$and $F^{-}$are given by the same expressions as $G^{+}$and $F^{+}$, with $\rho$ replaced by $1-\rho$ (that is, with $\theta$ changed to $-\theta$ ). By $\mathbb{P}^{x}$ and $\mathbb{E}^{x}$ we denote the probability and expectation corresponding to the process $X_{t}$ started at $x$. The following theorem is the first main result of the paper. Note also that $F^{+}$is continuous (cf . Lemma 3.1).

Theorem 1.1. Let $\tau_{0}$ be the first hitting time of $\{0\}$ for the process $X_{t}$. Then

$$
\mathbb{P}^{x}\left(\tau_{0}>t\right)=\frac{1}{\pi \cos \theta} \int_{0}^{\infty} \frac{e^{-s^{\alpha} t}}{s} F^{-}(s x) d s .
$$

for $x \neq 0$ and $t>0$.

The functions $F^{+}, F^{-}$can be seen as generalised eigenfunctions of transition operators of $X_{t}$ killed upon hitting $\{0\}$. These operators are defined by the formula

$$
P_{t}^{\mathbb{R} \backslash\{0\}} f(x)=\mathbb{E}^{x}\left(f\left(X_{t}\right) ; t<\tau_{0}\right),
$$

for $t>0, x \in \mathbb{R} \backslash\{0\}$, and they act on $\mathscr{L}^{p}(\mathbb{R} \backslash\{0\})$ for arbitrary $p \in[1, \infty]$. Although $P_{t}^{\mathbb{R} \backslash\{0\}} F^{-}$is not well-defined (the expectation does not converge), we have the following spectral-type representation of $P_{t}^{\mathbb{R} \backslash\{0\}}$. This is our second main result.

Theorem 1.2. There is a class of functions $\mathcal{H}$, dense in $\mathscr{L}^{2}(\mathbb{R} \backslash\{0\})$, with the following property. If $f, g \in \mathcal{H}$, then

$$
\begin{aligned}
& \int_{-\infty}^{\infty} P_{t}^{\mathbb{R} \backslash\{0\}} f(x) g(x) d x=\int_{0}^{\infty} \frac{e^{-s^{\alpha} t}}{\cos \theta}\left(\int_{-\infty}^{\infty} F^{+}(s x) f(x) d x\right)\left(\int_{-\infty}^{\infty} F^{-}(s y) g(y) d y\right) d s \\
& +\int_{0}^{\infty} \frac{e^{-s^{\alpha} t}}{\cos \theta}\left(\int_{-\infty}^{\infty} e^{-s x \sin \theta} \sin (s x \cos \theta) f(x) d x\right)\left(\int_{-\infty}^{\infty} e^{s y \sin \theta} \sin (s y \cos \theta) g(y) d y\right) d s .
\end{aligned}
$$

The class $\mathcal{H}$ is discussed in detail in Section 2.2. Theorem 1.2 provides a spectral-type representation of $P_{t}^{0}$ : the parenthesised integrals can be thought of as Fourier-type transforms of $f$ and $g$, which diagonalise the action of $P_{t}^{0}$. This is the reason we call $F^{+}$ and $F^{-}$generalised eigenfunctions of $P_{t}^{0}$. We stress, however, that due to exponential growth at infinity, $P_{t}^{0} F^{+}$is not defined unless the process $X_{t}$ is symmetric, that is, $\theta=0$. For the same reason we cannot write

$$
\int_{-\infty}^{\infty} P_{t}^{\mathbb{R} \backslash\{0\}} f(x) F^{+}(s x) d x=e^{-s^{\alpha} t} \int_{-\infty}^{\infty} f(x) F^{+}(s x) d x,
$$

$t>0, s>0$, even for $f \in \mathcal{H}$. The left-hand side is usually not well-defined.

We mention here one property of the functions $G^{+}$and $G^{-}$. For further information, see Section 3.

Proposition 1.3. The functions $G^{+}$and $G^{-}$are bounded, integrable, and their Fourier transform is given by

$$
\mathscr{F} G^{+}(\xi)=\mathscr{F} G^{-}(-\xi)=\sin \frac{\pi}{\alpha}\left(\frac{\alpha}{\psi(\xi)-1}-\frac{1}{e^{-i \theta} \xi-1}+\frac{1}{e^{i \theta} \xi+1}\right), \quad \xi \in \mathbb{R} ;
$$

Furthermore, the functions $G^{+}(x)=G^{-}(-x)$ and $G^{+}(-x)=G^{-}(x)$ are completely monotone on $(0, \infty)$. 
Remark 1.4. For $\theta=0$, i.e. the symmetric case, we reproduce the result of Example 5.1 in [23]. In this case function $G^{+}=G^{-}$takes form

$$
G^{+}(s x)=\frac{\alpha s^{\alpha-1} \sin \frac{\pi \alpha}{2} \sin \frac{\pi}{\alpha}}{\pi} \int_{0}^{\infty} \frac{t^{\alpha}}{1-2 t^{\alpha} \cos (\pi \alpha)+t^{2 \alpha}} e^{-s|x| t} d t,
$$

$F^{+}=F^{-}$and

$$
F^{+}(s x)=\sin \left(|s x|+\frac{\pi}{\alpha}-\frac{\pi}{2}\right)-G^{+}(s x) .
$$

\subsection{Structure of the article}

The remainning part of the paper is divided into four sections. In Preliminaries we recall basic definitions and state auxiliary lemmas. In particular, we introduce a suitable family of test functions $\mathcal{H}$, and we discuss Nevanlinna class of functions and Cauchy's integral formula. In Section 3 we prove a handful of technical lemmas in order to derive a formula for the generalised eigenfunctions $F^{+}, F^{-}$. The properties of functions $G^{+}$ and $G^{-}$are studied here, and the proof of Proposition 1.3 is given. Section 4 is dedicated to the proof of the Theorem 1.2 and, finally, Theorem 1.1 is proved in Section 5.

In our proof, we deform the contour of integration a number of times. Here is a rough sketch of the argument.

- We begin with the triple integral $I(\lambda)$ of $e^{-\lambda t} p_{t}^{0}(x, y) f(x) g(y)$.

- We use Plancherel's theorem to rewrite $I(\lambda)$ as a triple integral with respect to $t, \xi$, $\eta$, where $\xi$ and $\eta$ are the Fourier variables corresponding to $x$ and $y$ (Lemma 4.1).

- Next, we deform the contour of integration in $\xi$ and $\eta$ to $\left(-e^{-i \theta} \infty, 0\right) \cup\left(0, e^{i \theta} \infty\right)$.

- By doing so, we obtain an expression for $I(\lambda)$, which is a Cauchy-Stieltjes transform $\phi_{4}(\lambda)$ of some function of a new variable $r$ (Lemma 4.2).

- Since the Cauchy-Stieltjes transform $(r \mapsto \lambda)$ is the Laplace transform $(t \mapsto \lambda)$ of the Laplace transform $(r \mapsto t)$, the above leads to an expression for the double integral $J(t)$ of $p_{t}^{0}(x, y) f(x) g(y)$ (with respect to $x$ and $y$ ) as a Laplace transform of what will be denoted by $-\operatorname{Im} \phi_{4}(-r)$ (Theorem 4.7).

- In order to prove Theorem 1.2, we now identify the expression for $-\operatorname{Im} \phi_{4}(-r)$ (which is given in terms of integrals of Laplace transforms of $f$ and $g$ ) with an appropriate integral transform of $f$ and $g$. This involves deforming back the contour of integration with respect to $\xi$ and $\eta$ to $\mathbb{R}$, and an application of Plancherel's formula. It is here convenient to replace $r$ with $s^{\alpha}$.

- Theorem 1.1 is proved in a similar way, with an additional step at the end of the proof: we change the order of the integrals with respect to $s$ and $x$, and by a density argument, we are able to remove the integral with respect to $x$. Changing the order of integration, however, is not straightforward: it requires an appropriated deformation of the contour of integration, so that Fubini's theorem can be applied.

\section{Preliminaries}

We denote by $\mathscr{L}^{p}(\mathbb{R})$ the space of real-valued functions $f$ on $\mathbb{R}$ such that $|f(x)|^{p}$ is integrable. We use $\mathscr{L} f$ to denote the two-sided Laplace transform of $f$ :

$$
\mathscr{L} f(\xi)=\int_{-\infty}^{\infty} f(x) e^{-\xi x} d x
$$


whenever the integral converges absolutely. If $f \in \mathscr{L}^{1}(\mathbb{R})$, then $\mathscr{F} f(\xi)=\mathscr{L} f(i \xi)$ (with $\xi \in \mathbb{R}$ ) is the Fourier transform of $f$. The Fourier transformation $\mathscr{F}$ is extended continuously to $\mathscr{L}^{2}(\mathbb{R})$.

\subsection{Stable Lévy processes}

By $X_{t}$ we denote a one-dimensional $\alpha$-stable Lévy process with index of stability $\alpha \in(1,2)$. We assume that $\alpha>1$ in order that $X_{t}$ is point-recurrent (i.e. it hits single points with positive probability). The case $\alpha=2$ is well-studied and much simpler, so we require that $\alpha \neq 2$.

A one-dimensional stable Lévy process is completely characterised by $\alpha$, the positivity parameter $\rho=\mathbb{P}^{0}\left(X_{1}>0\right)$, and the scale parameter $k>0$. For $\alpha \in(1,2)$, we have $\rho \in\left[1-\frac{1}{\alpha}, \frac{1}{\alpha}\right]$. We denote by $\psi$ the characteristic exponent of $X_{t}$ :

$$
\mathbb{E}^{0} e^{i \xi X_{t}}=e^{-t \psi(\xi)}, t>0, \xi \in \mathbb{R}
$$

In our case

$$
\psi(\xi)=(k|\xi|)^{\alpha}\left(1-i \tan \left((2 \rho-1) \frac{\alpha \pi}{2}\right) \operatorname{sign} \xi\right), \quad \xi \in \mathbb{R} .
$$

Our results do not depend on the scale parameter $k$ in any essential way. For this reason, we choose $k$ in such a way that $|\psi(\xi)|=|\xi|^{\alpha}$ for every $\xi \in \mathbb{R}$. Thus, if we set

$$
\theta=\frac{(2 \rho-1) \pi}{2}
$$

then we have $|\theta| \leqslant \frac{\pi}{\alpha}-\frac{\pi}{2}$ and

$$
\psi(\xi)= \begin{cases}\left(e^{-i \theta} \xi\right)^{\alpha} & \text { if } \xi>0, \\ \left(e^{i \theta}(-\xi)\right)^{\alpha} & \text { if } \xi<0\end{cases}
$$

(all complex powers are principal branches). Note that if we replace conditions $\xi>0$, $\xi<0$ by $\operatorname{Re} \xi>0, \operatorname{Re} \xi<0$, respectively, then the above expression defines a analytic extension of $\psi$ to $\mathbb{C} \backslash i \mathbb{R}$. Throughout the text, the symbol $\psi$ denotes this extension, and the fact that $\psi\left(r e^{i \theta}\right) \in(0, \infty)$ for $r \in(0, \infty)$ will play an important role.

Remark 2.1. Apparently, the results of the present article can be extended to some Lévy processes with completely monotone jumps, introduced in [37] and studied recently in [25]. For symmetric Lévy processes this was already done in [23]. In the nonsymmetric case, one clearly has to assume that $1 /(1+\psi(\xi))$ is absolutely integrable, so that $X_{t}$ is point-recurrent, and points are regular for $X_{t}$ (see [41], Theorem 43.3). However, a number of further technical conditions will have to be imposed.

Probability and expectation of the process starting from $x \in \mathbb{R}$ are denoted by $\mathbb{P}^{x}$ and $\mathbb{E}^{x}$. We define the transition operators $P_{t}$ of $X_{t}$ by

$$
P_{t} f(x)=\mathbb{E}^{x} f\left(X_{t}\right)=\int_{\mathbb{R}} f(y) \mathbb{P}^{x}\left(X_{t} \in d y\right), \quad t>0, x \in \mathbb{R} .
$$

The operators $P_{t}$ are convolution operators, and the corresponding convolution kernels $p_{t}(x)$ are known as transition densities. More formally, $p_{t}$ is such that $P_{t} f(x)=$ $\int_{\mathbb{R}} f(y) p_{t}(x-y) d y$. The operators $P_{t}$ form a strongly continuous semigroup of operators on $\mathscr{L}^{2}(\mathbb{R})$, and their action is diagonalised by the Fourier transformation.

Let $D \subseteq \mathbb{R}$ be an open set and let

$$
\tau_{D}=\inf \left\{t \geqslant 0: X_{t} \notin D\right\}
$$


be the first exit time of the process $X_{t}$ from the set $D$. The process $X_{t}$ killed upon leaving $D$ is formally defined to be equal to $X_{t}$ until its life-time $\tau_{D}$. We are more interested in the corresponding transition operators $P_{t}^{D}$, given by

$$
P_{t}^{D} f(x)=\mathbb{E}^{x}\left(f\left(X_{t}\right) \chi_{t<\tau_{D}}\right)=\int_{D} f(y) \mathbb{P}^{x}\left(X_{t} \in d y ; t<\tau_{D}\right), \quad t>0, x \in D .
$$

The corresponding kernel function $p_{t}^{D}(x, y)$, the transition density of the killed process, is given by so called Dynkin-Hunt formula (we use the name from [8]; it is a consequence of strong Markov property and it was used in similar context in [45]; the proof for the Brownian motion case can be found in [1], Section II.4, and in [9], Section 2.2, however these proofs are general and can be used in our case)

$$
p_{t}^{D}(x, y)=p_{t}(y-x)-\mathbb{E}^{x}\left(p_{t-\tau_{D}}\left(y-X_{\tau_{D}}\right) \chi_{t>\tau_{D}}\right), \quad t>0, x, y \in D .
$$

In particular, $0 \leqslant p_{t}^{D}(x, y) \leqslant p_{t}(y-x)$. We consider $D=\mathbb{R} \backslash\{0\}$, and for simplicity we denote $\tau_{0}=\tau_{\mathbb{R} \backslash\{0\}}, P_{t}^{0}=P_{t}^{\mathbb{R} \backslash\{0\}}$ and $p_{t}^{0}(x, y)=p_{t}^{\mathbb{R} \backslash\{0\}}(x, y)$.

Recall that the $\lambda$-potential kernel of the process $X_{t}$ is defined as

$$
u_{\lambda}(x)=\int_{0}^{\infty} e^{-\lambda t} p_{t}(x) d t, \quad \lambda>0, x \in \mathbb{R} .
$$

By (2.1) and Fubini's theorem, we have

$$
\mathscr{F} p_{t}(\xi)=e^{-t \psi(-\xi)}, \quad \mathscr{F} u_{\lambda}(\xi)=\frac{1}{\psi(-\xi)+\lambda}, \quad \xi \in \mathbb{R} .
$$

In a similar way, the $\lambda$-potential kernel of the killed process is given by

$$
u_{\lambda}^{0}(x, y)=\int_{0}^{\infty} e^{-\lambda t} p_{t}^{0}(x, y) d t, \quad \lambda>0, x, y \in \mathbb{R} .
$$

We have

$$
u_{\lambda}^{0}(x, y)=u_{\lambda}(y-x)-\frac{u_{\lambda}(-x) u_{\lambda}(y)}{u_{\lambda}(0)} .
$$

This well-known identity follows from Dynkin-Hunt formula by integration, as follows. Observe that:

$$
\begin{aligned}
u_{\lambda}^{0}(x, y) & =\int_{0}^{\infty} e^{-\lambda t} p_{t}^{0}(x, y) d t=\int_{0}^{\infty} e^{-\lambda t} p_{t}(x, y) d t-\mathbb{E}^{x} \int_{\tau_{0}}^{\infty} e^{-\lambda t} p_{t-\tau_{0}}\left(y-X_{\tau_{0}}\right) d t \\
& =u_{\lambda}(y-x)-\mathbb{E}^{x} \int_{0}^{\infty} e^{-\lambda s-\lambda \tau_{0}} p_{s}(y-0) d s=u_{\lambda}(y-x)-\mathbb{E}^{x} e^{-\lambda \tau_{0}} u_{\lambda}(y) .
\end{aligned}
$$

By Theorem 43.3 (4) in [41],

$$
\mathbb{E}^{x} e^{-\lambda \tau_{0}}=\frac{u_{\lambda}(-x)}{u_{\lambda}(0)}
$$

and (2.2) follows.

Our ultimate goal is to find integral expressions for the distribution of $\tau_{0}$ with respect to $\mathbb{P}^{x}$. Our starting points are (2.2) and (2.4), which describe the Laplace transforms (with respect to $t$ ) of $p_{t}^{0}(x, y)$ and $\mathbb{P}^{x}\left(\tau_{0} \in d t\right)$ in terms of $u_{\lambda}$. The function $u_{\lambda}$ is in turn the inverse Fourier transform of $1 /(\lambda+\psi(-\xi))$. These expressions, however, are not suitable for standard inversion formulas. For this reason, we will first multiply the above expressions by appropriately regular test functions, and only then consider Fourier transforms in $x$ and $y$. 


\subsection{Test functions}

Just as in the symmetric case studied in [23], our representation of $p_{t}^{0}(x, y)$ and $\mathbb{P}^{x}\left(\tau_{0}>t\right)$ involves generalised eigenfunctions $F^{+}$and $F^{-}$. In the symmetric case, $F^{+}=F^{-}$is a bounded function; here $F^{+}$and $F^{-}$have exponential growth at infinity. This nuisance makes the use of the Laplace transform problematic. To overcome this difficulty, we introduce a particular class of test functions, following [21] (where stable processes in a half-line were studied).

Definition 2.2. By $\mathcal{H}_{+}$denote the class of all functions $g: \mathbb{R} \rightarrow \mathbb{R}$ such that

1. $g(x)=0$ for $x<0$;

2. $g$ extends to an analytic function in the sector $|\arg (z)|<\pi / 2$;

3. for every $\varepsilon \in(0, \pi / 2)$ there exists $\delta=\delta(\varepsilon)>0$ such that $|g(z)|=O\left(|z|^{-\delta|z|}\right)$ when $|z| \rightarrow \infty$, and $|g(z)|=O(1)$ when $|z| \rightarrow 0$, uniformly in the sector $|\arg (z)|<\pi / 2-\varepsilon$.

We say that $g \in \mathcal{H}_{-}$if $g(-z)$ belongs to $\mathcal{H}_{+}$. Finally, $g \in \mathcal{H}$ if $g: \mathbb{R} \rightarrow \mathbb{R}$, and $g=g_{+}+g_{-}$ for some $g_{+} \in \mathcal{H}_{+}$and $g_{-} \in \mathcal{H}_{-}$.

We note that the classes $\mathcal{H}_{+}, \mathcal{H}_{-}, \mathcal{H}$ are non-empty and non-trivial, e.g. $g(z)=$ $e^{-s|z| \log |z+e|}$ is in $\mathcal{H}$ for every $s>0$. It was observed in [21] that, on one hand, $\mathcal{H}_{+}$is sufficiently rich, while on the other one, the Laplace-Fourier transform of a function $g \in \mathcal{H}_{+}$is a suitably decaying analytical function in the sector $|\operatorname{Arg}(z)|<\pi-\varepsilon$ for every $\varepsilon>0$. We rephrase these results for the class $\mathcal{H}$.

Lemma 2.3 ([21], p. 19, Lemma 2.14). Let $g \in \mathcal{H}$. Then $\mathscr{L} g(z)$ is an entire function and for every $\varepsilon \in\left(0, \frac{\pi}{2}\right)$ there exists a constant $C$ such that

$$
|\mathscr{L} g(z)| \leqslant C \min \left\{1,|z|^{-1}\right\}, \quad|\arg (i z)| \leqslant \frac{\pi}{2}-\varepsilon \text { or }|\arg (-i z)| \leqslant \frac{\pi}{2}-\varepsilon .
$$

A similar argument leads to the following estimate of the derivative of $\mathscr{L} g$; we omit the proof.

Lemma 2.4. Let $g \in \mathcal{H}$. Then for every $\varepsilon \in\left(0, \frac{\pi}{2}\right)$ there exists a constant $C$ such that

$$
\left|(\mathscr{L} g)^{\prime}(z)\right| \leqslant C \min \left\{1,|z|^{-2}\right\}, \quad|\arg (i z)| \leqslant \frac{\pi}{2}-\varepsilon \text { or }|\arg (-i z)| \leqslant \frac{\pi}{2}-\varepsilon .
$$

Lemma 2.5. The class $\mathcal{H}$ is dense in the following sense: if $f$ is a Borel-measurable function and

$$
\int_{-\infty}^{\infty} f(x) g(x) d x=0 \quad \text { for every } g \in \mathcal{H},
$$

with the integral absolutely convergent, then $f(x)=0$ for almost every $x \in \mathbb{R}$.

Proof. Let $\rho(x)=x \log (x+e)$ and consider $g(x)=e^{-s \rho(x)} \chi_{(0, \infty)}(x)$ for some $s>0$. As we already remarked above, $g \in \mathcal{H}$ : it extends to an analytic function in the sector $|\arg (z)|<\frac{\pi}{2}$, and we have $|g(z)|=|z+e|^{-s|z|}=O\left(|z|^{-s|z| / 2}\right)$ as $|z| \rightarrow \infty$ and $|g(z)|=O(1)$ as $|z| \rightarrow 0$, uniformly in the sector $|\arg z| \leqslant \frac{\pi}{2}-\epsilon$ for every $\epsilon>0$. Note that $\rho^{\prime}(x)>0$ and, by substitution $y=\rho(x)$, we get

$$
0=\int_{-\infty}^{\infty} f(x) g(x) d x=\int_{0}^{\infty} e^{-s y} f\left(\rho^{-1}(y)\right)\left(\rho^{-1}\right)^{\prime}(y) d y
$$

for every $s>0$. This means that the Laplace transform of $f\left(\rho^{-1}(y)\right)\left(\rho^{-1}\right)^{\prime}(y)$ vanishes for every $s>0$, and hence $f\left(\rho^{-1}(y)\right)\left(\rho^{-1}\right)^{\prime}(y)=0$ for almost every $y>0$. It follows that $f(x)=0$ for almost every $x>0$. A very similar argument shows that $f(x)=0$ for almost every $x<0$, and the proof is complete. 


\subsection{Stieltjes functions and Cauchy's integral formula for the upper half-plane}

One of the key steps in the proof of our main result involves Stieltjes-type representation of analytic functions in $\mathbb{C} \backslash(-\infty, 0)$. We deduce this result from Cauchy's integral formula for functions from the Hardy space $\mathscr{H}^{p}$ in the upper complex half-plane $H_{+}=\{z \in \mathbb{C}: \operatorname{Im} z>0\}$. We begin with a number of standard definitions.

Definition 2.6. A function $f:(0, \infty) \rightarrow \mathbb{R}$ is a Stieltjes function if

$$
f(x)=\frac{c_{1}}{x}+c_{2}+\frac{1}{\pi} \int_{0}^{\infty} \frac{m(d s)}{x+s}, \quad x>0,
$$

where $c_{1}, c_{2} \geqslant 0$ and $m$ is a non-negative Radon measure on $(0, \infty)$ which satisfies the integrability condition $\int_{0}^{\infty} \min \left\{1, s^{-1}\right\} m(d s)<\infty$.

Definition 2.7. For $p \in(1, \infty)$, by $\mathscr{H}^{p}$ we denote the space of functions $f$ analytic in $H_{+}$such that $|f(x+i y)|^{p}$ is integrable with respect to $x \in \mathbb{R}$ for each $y>0$, and $\int_{\mathbb{R}}|f(x+i y)|^{p} d x$ is a bounded function of $y>0$.

Definition 2.8. A function $f$ analytic in $H_{+}$is an outer function if

$$
\log |f(x+i y)|=\frac{1}{\pi} \int_{-\infty}^{\infty} \frac{y}{(x-s)^{2}+y^{2}} \log |f(s)| d s
$$

for every $x \in \mathbb{R}$ and $y>0$. Here for $s \in \mathbb{R}$ the symbol $f(s)$ denotes the limit $\lim _{t \rightarrow 0^{+}} f(s+$ $i t)$, which necessarily exists for almost every $s \in \mathbb{R}$.

Definition 2.9. The Nevanlinna class $\mathscr{N}^{+}$is the set of functions $f$ analytic in $H_{+}$, which can be represented as $f=f_{1} / f_{2}$, where $f_{1}$ and $f_{2}$ are analytic and bounded in $H_{+}$, and $f_{2}$ is outer.

The class of outer functions on the unit disk, and the Nevanlinna class $\mathscr{N}^{+}$on the unit disk, are defined in a similar way. Note that composition with a conformal map between the unit disk and the upper complex half-plane defines a bijection between the corresponding classes of outer functions, as well as between the corresponding Nevanlinna classes $\mathscr{N}^{+}$. We refer to [6, 39] for further details.

We will use the following standard properties of Stieltjes functions, Hardy space $\mathscr{H}^{p}$, and the Nevanlinna class $\mathscr{N}^{+}$.

Lemma 2.10 ([23], Proposition 2.1(b)). A function $f:(0, \infty) \rightarrow \mathbb{R}$ is a Stieltjes function if and only if $f(z) \geqslant 0$ for $z>0$ and either $f$ is constant, or $f$ extends to a analytic function in $\mathbb{C} \backslash(-\infty, 0]$, which swaps the upper and the lower complex half-planes, i.e. if $\operatorname{Im} z>0$, then $\operatorname{Im} f(z)<0$ and if $\operatorname{Im} z<0$, then $\operatorname{Im} f(z)>0$.

Lemma 2.11 ([39], Theorem 4.29). If $f$ is an analytic function in the unit disk with non-negative real part and $f$ is not 0 everywhere, then $f$ is an outer function.

Lemma 2.12. If $f$ is an analytic function in $H_{+}, \operatorname{Re} f \geqslant 0$ in $H_{+}$and $f$ is not 0 everywhere, then $f$ is an outer function. If $f$ is a Stieltjes function, then (the analytic extension of) $f$ is an outer function.

Proof. Suppose that $\operatorname{Re} f \geqslant 0$ in $H_{+}$, and consider the conformal map $w(z)=-i(z-$ 1)/( $z+1)$ from the unit disk $\{z \in \mathbb{C}:|z|<1\}$ onto the upper complex half-plane $H_{+}$. If $g(z)=f(w(z))$ for $z \in B_{1}$, then $g$ is an analytic function in the unit disk, with nonnegative real part. By Lemma 2.11, $g$ is an outer function on the unit disk. It follows that $f(z)=-i g\left(w^{-1}(z)\right)$ is an outer function in the upper complex half-plane $H_{+}$.

If $f$ is the analytic extension to the upper complex half-plane $H_{+}$of a Stieltjes function, then, by Lemma 2.10, $\operatorname{Re}(i f(z)) \geqslant 0$ for every $z \in H_{+}$. By the first part of the proof, if is outer, and hence also $f$ is outer. 
Lemma 2.13 ([39], 5.14(iv)). The sum of functions from $\mathscr{N}^{+}$is in $\mathscr{N}^{+}$. The product of functions from $\mathscr{N}^{+}$is in $\mathscr{N}^{+}$. The ratio of a function in $\mathscr{N}^{+}$and an outer function is in $\mathscr{N}^{+}$.

Lemma 2.14. The function $f(z)=\sqrt{z}$ is outer.

Proof. The function $1 / f(z)=1 / \sqrt{z}$ is a Stieltjes function. Hence, $1 / f$ is outer, and it follows that $f$ is outer, too.

Recall that for $x \in \mathbb{R}$ limit $\lim _{z \rightarrow x} f(z)=a$ is said to exist non-tangentially if $\lim _{n \rightarrow \infty} f\left(z_{n}\right)=a$ whenever $\lim _{n \rightarrow \infty} z_{n}=x$ and there is a constant $c>0$ such that $\left|\operatorname{Re}\left(z_{n}-x\right)\right| \leqslant c \operatorname{Im} z_{n}$ for all $n \in \mathbb{N}$.

Lemma 2.15 ([39], Theorems 5.6 and 5.23(i)). Let $f$ be in the Nevanlinna class $\mathscr{N}^{+}$. Then the boundary limit $f(x)=\lim _{z \rightarrow x} f(z)$ exists non-tangentially for almost every $x \in \mathbb{R}$. Furthermore, if

$$
\int_{\mathbb{R}}|f(x)|^{p} d x<\infty
$$

for some $p \in(1, \infty)$, then $f \in \mathscr{H}^{p}$.

Theorem 2.16 ([11], Theorem 11.8). Let $f$ be in $\mathscr{H}^{p}$ for some $p \in(1, \infty)$. Then

$$
f(z)=\frac{1}{2 \pi i} \int_{\mathbb{R}} \frac{f(t)}{t-z} d t
$$

when $\operatorname{Im} z>0$ and

$$
0=\frac{1}{2 \pi i} \int_{\mathbb{R}} \frac{f(t)}{t-z} d t
$$

when $\operatorname{Im} z<0$.

Corollary 2.17. Let $f$ be an analytic function in $\mathbb{C} \backslash(-\infty, 0]$, which is real-valued on $(0, \infty)$, and such that $f\left(-\xi^{2}\right)$ belongs to the Nevanlinna class $\mathscr{N}^{+}$in the upper complex half-plane. For $s>0$ denote by $f(-s)$ the boundary $\operatorname{limit} \lim _{t \rightarrow 0^{+}} f(-s+i t)$ (which exists for almost every $s)$. Suppose that $\int_{0}^{\infty}\left|f\left(-s^{2}\right)\right|^{p} d s<\infty$ for some $p \in(1, \infty)$. Then

$$
f(z)=-\frac{1}{\pi} \int_{0}^{\infty} \frac{\operatorname{Im} f(-s)}{s+z} d s=\frac{1}{\pi} \int_{0}^{\infty} \frac{\sqrt{z}}{\sqrt{s}} \frac{\operatorname{Re} f(-s)}{s+z} d s, \quad z \in \mathbb{C} \backslash(-\infty, 0] .
$$

Proof. We define an auxiliary function $g(\xi)=f\left((-i \xi)^{2}\right)$ in the upper complex half-plane $\operatorname{Im} \xi>0$, and we verify that $g$ satisfies the assumptions of Theorem 2.16. By assumption, $g$ is in the Nevanlinna class $\mathscr{N}^{+}$, so $g$ has a non-tangential boundary limit almost everywhere.

Fix $s>0$. Let $H(t)=i \sqrt{-s+i t}$ for $t \geqslant 0$. Then $H^{\prime}(t)=-1 /(2 \sqrt{-s+i t})$, and hence $H^{\prime}(0)=-1 /(2 \sqrt{-s})=i /(2 \sqrt{s})$ is purely imaginary. This implies that $H(t)$ approaches $z(0)=i \sqrt{-s}=-\sqrt{s}$ non-tangentially in the upper complex half-plane. As a consequence (since $f(-s+i t)=g(i \sqrt{-s+i t})$ ), we have:

$$
f(-s):=\lim _{t \rightarrow 0^{+}} f(-s+i t)=\lim _{t \rightarrow 0^{+}} g(i \sqrt{-s+i t})=g(-\sqrt{s})
$$

for almost all $s>0$. Since $f(\bar{z})=\overline{f(z)}$, we find that $g(-\bar{z})=f\left((i \bar{z})^{2}\right)=f\left(-\bar{z}^{2}\right)=$ $\overline{f\left(-z^{2}\right)}=\overline{f\left((-i z)^{2}\right)}=\overline{g(z)}$ for $z \in\{z \in \mathbb{C}: \operatorname{Im} z>0\}$ and hence $g(s)=\overline{g(-s)}=f\left(-s^{2}\right)$ for almost all $s>0$.

By Lemma 2.15 and the assumption $\int_{0}^{\infty}\left|f\left(-s^{2}\right)\right|^{p} d s<\infty, g$ is in the Hardy space $\mathscr{H}^{p}$. Therefore, by Theorem 2.16, for $z \in \mathbb{C} \backslash(-\infty, 0]$ we have

$$
f(z)=g(i \sqrt{z})=\frac{1}{2 \pi i} \int_{-\infty}^{\infty} \frac{g(t)}{t-i \sqrt{z}} d t=\frac{1}{2 \pi i} \int_{0}^{\infty} \frac{\overline{f\left(-t^{2}\right)}}{t-i \sqrt{z}} d t-\frac{1}{2 \pi i} \int_{0}^{\infty} \frac{f\left(-t^{2}\right)}{t+i \sqrt{z}} d t,
$$


and, similarly,

$$
0=\frac{1}{2 \pi i} \int_{-\infty}^{\infty} \frac{g(t)}{t+i \sqrt{z}} d t=\frac{1}{2 \pi i} \int_{0}^{\infty} \frac{\overline{f\left(-t^{2}\right)}}{t+i \sqrt{z}} d t-\frac{1}{2 \pi i} \int_{0}^{\infty} \frac{f\left(-t^{2}\right)}{t-i \sqrt{z}} d t .
$$

Adding the corresponding sides of these identities, we find that

$$
\begin{aligned}
f(z) & =\frac{1}{2 \pi i} \int_{0}^{\infty}\left(\overline{f\left(-t^{2}\right)}-f\left(-t^{2}\right)\right)\left(\frac{1}{t-i \sqrt{z}}+\frac{1}{t+i \sqrt{z}}\right) d t \\
& =\frac{1}{2 \pi i} \int_{0}^{\infty}\left(-2 i \operatorname{Im} f\left(-t^{2}\right)\right) \frac{2 t}{t^{2}+z} d t=-\frac{1}{\pi} \int_{0}^{\infty} \frac{\operatorname{Im} f(-s)}{s+z} d s .
\end{aligned}
$$

as desired. Similarly, subtracting the corresponding sides rather than adding them, we obtain

$$
\begin{aligned}
f(z) & =\frac{1}{2 \pi i} \int_{0}^{\infty}\left(\overline{f\left(-t^{2}\right)}+f\left(-t^{2}\right)\right)\left(\frac{1}{t-i \sqrt{z}}-\frac{1}{t+i \sqrt{z}}\right) d t \\
& =\frac{1}{2 \pi i} \int_{0}^{\infty}\left(2 \operatorname{Re} f\left(-t^{2}\right)\right) \frac{2 i \sqrt{z}}{t^{2}+z} d t=\frac{1}{\pi} \int_{0}^{\infty} \frac{\sqrt{z}}{\sqrt{s}} \frac{\operatorname{Re} f(-s)}{s+z} d s
\end{aligned}
$$

as desired.

\subsection{Auxiliary lemmas}

We need the following simple corollary of the residue theorem.

Lemma 2.18 ([21], p. 11, Lemma 2.5). Let $f$ be an analytic function in the sector $-\epsilon<\operatorname{Arg}(z)<b+\epsilon(b-\epsilon<\operatorname{Arg} z<\epsilon)$ for some $\epsilon>0$ and $b>0$ (resp. $b<0$ ), except for a finite number of poles at points $z=z_{j}$ lying in the sector $0<\operatorname{Arg}(z)<b$ (resp. $0>\operatorname{Arg}(z)>b)$. Assume also that for some $\delta>0$ we have $f(z)=O\left(|z|^{-1+\delta}\right)$ as $|z| \rightarrow 0^{+}$ and $f(z)=O\left(|z|^{-1-\delta}\right.$ ) as $|z| \rightarrow+\infty$, uniformly in the sector $0 \leqslant \operatorname{Arg}(z) \leqslant b$ (resp. $0 \geqslant \operatorname{Arg}(z) \geqslant b)$. Then

$$
\int_{0}^{\infty} f(z) d z=e^{i b} \int_{0}^{\infty} f\left(e^{i b} z\right) d z+2 \pi i \sum_{j} \operatorname{Res}\left(f\left(z_{j}\right)\right)
$$

(resp. $\int_{0}^{\infty} f(z) d z=e^{i b} \int_{0}^{\infty} f\left(e^{i b} z\right) d z-2 \pi i \sum_{j} \operatorname{Res}\left(f\left(z_{j}\right)\right)$ for the case $b<0$ ).

We will need the following technical estimate.

Lemma 2.19. Suppose that $\alpha \in(1,2)$ and $h:(0, \infty) \rightarrow \mathbb{R}$ satisfies $|h(r)| \leqslant c_{1} \min \left\{1, r^{-1}\right\}$ and $\left|h^{\prime}(r)\right| \leqslant c_{2} \min \left\{1, r^{-2}\right\}$ if $r>0$. Let

$$
K(s)=\text { p.v. } \int_{0}^{\infty} \frac{h(r)}{r^{\alpha}-s^{\alpha}} d r, \quad s>0 .
$$

Then there is a constant $C$ (which depends only on $\alpha, c_{1}$ and $c_{2}$ ) such that

$$
|K(s)| \leqslant \begin{cases}C s^{1-\alpha} & \text { if } 0<s<1 \\ C s^{-\alpha} \log (1+s) & \text { if } s \geqslant 1 .\end{cases}
$$

Proof. Fix $s>0$. Since $\left|r^{\alpha}-s^{\alpha}\right| \geqslant r^{\alpha}-\left(\frac{1}{2} r\right)^{\alpha} \geqslant \frac{1}{2} r^{\alpha}$ if $r \geqslant 2 s$, we have

$$
|K(s)| \leqslant\left|\int_{0}^{2 s} \frac{h(r)-h(s)}{r^{\alpha}-s^{\alpha}} d r\right|+\mid h(s) \text { p.v. } \int_{0}^{2 s} \frac{1}{r^{\alpha}-s^{\alpha}} d r \mid+2 \int_{2 s}^{\infty} \frac{|h(r)|}{r^{\alpha}} d r .
$$

The third integral in the right-hand side is easy to estimate: if $2 s \geqslant 1$, we have

$$
\int_{2 s}^{\infty} \frac{|h(r)|}{r^{\alpha}} d r \leqslant c_{1} \int_{2 s}^{\infty} \frac{1}{r^{\alpha+1}} d r=\frac{c_{1}}{\alpha(2 s)^{\alpha}},
$$


while if $0<2 s<1$,

$$
\int_{2 s}^{\infty} \frac{|h(r)|}{r^{\alpha}} d r \leqslant c_{1} \int_{2 s}^{\infty} \frac{1}{r^{\alpha}} d r=\frac{c_{1}}{(\alpha-1)(2 s)^{\alpha-1}} .
$$

The middle integral in (2.13) also shows no difficulties:

$$
\mid h(s) \text { p.v. } \int_{0}^{2 s} \frac{1}{r^{\alpha}-s^{\alpha}} d r\left|\leqslant c_{1} \min \left\{1, s^{-1}\right\} \times s^{1-\alpha}\right| \text { p.v. } \int_{0}^{2} \frac{1}{t^{\alpha}-1} d t \mid .
$$

We will show that the principal value p.v. $\int_{0}^{2} \frac{1}{t^{\alpha}-1} d t$ is well-defined. Write

$$
\frac{1}{t^{\alpha}-1}=\frac{1}{\alpha} \frac{1}{t-1}+\frac{\alpha(t-1)-\left(t^{\alpha}-1\right)}{\alpha(t-1)\left(t^{\alpha}-1\right)}
$$

The first term does not cause any problems and the second term extends continuously at 1 by l'Hôspital's rule applied twice:

$$
\lim _{t \rightarrow 1} \frac{\alpha(t-1)-\left(t^{\alpha}-1\right)}{\alpha(t-1)\left(t^{\alpha}-1\right)}=\lim _{t \rightarrow 1} \frac{1-t^{\alpha-1}}{\alpha(t-1) t^{\alpha-1}+t^{\alpha}-1}=\lim _{t \rightarrow 1} \frac{(1-\alpha) t^{\alpha-2}}{2 \alpha t^{\alpha-1}}=\frac{1-\alpha}{2 \alpha} .
$$

The estimate of the first integral in (2.13) requires more work. Since

$$
\left|h^{\prime}(t)\right| \leqslant c_{2} \min \left\{1, t^{-2}\right\} \leqslant 2 c_{2}(1+t)^{-2},
$$

we have

$$
|h(r)-h(s)|=\left|\int_{s}^{r} h^{\prime}(t) d t\right| \leqslant 2 c_{2}\left|\frac{1}{1+s}-\frac{1}{1+r}\right|=\frac{2 c_{2}|r-s|}{(1+r)(1+s)} .
$$

Therefore,

$$
\left|\int_{0}^{2 s} \frac{h(r)-h(s)}{r^{\alpha}-s^{\alpha}} d r\right| \leqslant 2 c_{2} \int_{0}^{2 s} \frac{r-s}{r^{\alpha}-s^{\alpha}} \frac{1}{(1+r)(1+s)} d r .
$$

Since $(t-1) /\left(t^{\alpha}-1\right) \leqslant 1$ for all $t>0$, we have

$$
\frac{r-s}{r^{\alpha}-s^{\alpha}}=\frac{1}{s^{\alpha-1}} \frac{(r / s)-1}{(r / s)^{\alpha}-1} \leqslant \frac{1}{s^{\alpha-1}} .
$$

It follows that

$$
\left|\int_{0}^{2 s} \frac{h(r)-h(s)}{r^{\alpha}-s^{\alpha}} d r\right| \leqslant \frac{2 c_{2}}{s^{\alpha-1}} \int_{0}^{2 s} \frac{1}{(1+r)(1+s)} d r=\frac{2 c_{2} \log (1+2 s)}{s^{\alpha-1}(1+s)} .
$$

Combining the above estimates, we conclude that

$$
K(s) \leqslant \frac{C_{1} \log (1+2 s)}{s^{\alpha-1}(1+s)}+C_{2} s^{1-\alpha} \min \left\{1, s^{-1}\right\}+C_{3} \min \left\{s^{-\alpha}, s^{1-\alpha}\right\}
$$

for some constants $C_{1}, C_{2}, C_{3}$. The desired result follows.

The following identity is quite elementary.

Lemma 2.20 ([23], equation (4.1)). For every $a, b, c \in \mathbb{C}, \operatorname{Im} c \neq 0$, we have

$$
\operatorname{Im}\left(\frac{a b}{c}\right)=\frac{\operatorname{Im} a \operatorname{Im} b}{\operatorname{Im} c}+\frac{\operatorname{Im}(a / c) \operatorname{Im}(b / c)}{\operatorname{Im}(1 / c)} .
$$

We need one more technical result. 
Lemma 2.21. Let $t>0$ and

$$
\Phi(z)=\int_{0}^{\infty} e^{-s^{\alpha} t} \frac{1-e^{-s z}}{s} d s
$$

for $z \in \mathbb{C}$. Then $\Phi$ is an entire function, and for every $\epsilon>0$ there is $C>0$ such that

$$
\left|\int_{0}^{\infty} e^{-s^{\alpha} t} \frac{e^{-s z_{2}}-e^{-s z_{1}}}{s} d s\right|=\left|\Phi\left(z_{1}\right)-\Phi\left(z_{2}\right)\right| \leqslant C
$$

whenever $\left|z_{1}\right|=\left|z_{2}\right|$ and both $z_{1}$ and $z_{2}$ are in the sector $|\operatorname{Arg} z| \leqslant \frac{\pi}{2}+\frac{\pi}{2 \alpha}-\epsilon$.

Proof. By Lemma 2.14 in [21] applied to the function $\exp \left(-s^{\alpha} t\right)$, the function

$$
\Psi(z)=\int_{0}^{\infty} e^{-s^{\alpha} t} e^{-s z} d s
$$

is entire, and for every $\epsilon>0$ there is $C>0$ such that if $|\operatorname{Arg} z| \leqslant \frac{\pi}{2}+\frac{\pi}{2 \alpha}-\epsilon$, then $|\Psi(z)| \leqslant C \min \left\{1,|z|^{-1}\right\}$. Integrating $\Psi$ over $[0, z]$ and using Fubini's theorem, we find that

$$
\int_{[0, z]} \Psi(w) d w=\int_{0}^{\infty} e^{-s^{\alpha} t} \int_{[0, z]} e^{-s w} d w d s=\int_{0}^{\infty} e^{-s^{\alpha} t} \frac{e^{-s z}-1}{-s} d s=\Phi(z) ;
$$

the use of Fubini's theorem is justified by the estimate $\left|e^{-s^{\alpha} t} e^{-s w}\right| \leqslant e^{-s^{\alpha} t} e^{s|z|}$. Therefore, $\Phi$ is indeed an entire function.

Suppose that $\varepsilon>0, r>0$ and that $z_{1}=r e^{i s_{1}}$ and $z_{2}=r e^{i s_{2}}$ are in the sector $|\operatorname{Arg} z| \leqslant \frac{\pi}{2}+\frac{\pi}{2 \alpha}-\epsilon$. Integrating over the arc $\Gamma$ of the circle $|z|=r$ with endpoints $z_{1}$ and $z_{2}$, we find that

$$
\left|\Phi\left(z_{2}\right)-\Phi\left(z_{1}\right)\right|=\left|\int_{\Gamma} \Psi(w) d w\right| \leqslant \int_{s_{1}}^{s_{2}}\left|\Psi\left(r e^{i s}\right)\right| r d s \leqslant 2 \pi r \times C \min \left\{1, r^{-1}\right\},
$$

which completes the proof.

\section{Properties of the function $G^{+}$}

Recall that the characteristic exponent of $X_{t}$ is given by $\psi(\xi)=\left(e^{-i \theta} \xi\right)^{\alpha}$ when $\xi>0$ and $\psi(\xi)=\left(-e^{i \theta} \xi\right)^{\alpha}$ when $\xi<0$, and these expressions extend analytically to $\operatorname{Re} \xi>0$ and $\operatorname{Re} \xi<0$, respectively.

Proof of Proposition 1.3. In terms of the characteristic exponent $\psi$, we need to prove that

$$
\mathscr{F} G^{+}(\xi)=\sin \frac{\pi}{\alpha}\left(\frac{\alpha}{\psi(\xi)-1}-\frac{1}{e^{-i \theta} \xi-1}+\frac{1}{e^{i \theta} \xi+1}\right), \quad \xi \in \mathbb{R} .
$$

One way to prove the above identity is to simply evaluate the left-hand side using the definition (1.1) of $G^{+}$. We take a different approach: we apply the inverse Fourier transform to the right-hand side of (3.1) and in this way we derive (1.1).

We denote the right-hand side of (3.1) by $\Phi(i \xi)$. Note that $|\Phi(i \xi)| \leqslant C \min \left\{1,|\xi|^{-1}\right\}$. In particular, $\Phi(i \xi)$ is square integrable, and hence $\Phi(i \xi)$ indeed is the Fourier transform of a function $G \in \mathscr{L}^{2}(\mathbb{R})$. Observe that $\Phi(-i \xi)=\overline{\Phi(i \xi)}$ for $\xi \in \mathbb{R}$, and hence $G$ is real-valued.

By definition, $\Phi(i \xi)$ given by right-hand side of (3.1), and hence $\Phi$ extends to an analytic function in the upper complex half-plane, continuous on the boundary, given by the formula

$$
\Phi(\xi)=\sin \frac{\pi}{\alpha}\left(\frac{\alpha}{\left(-i e^{-i \theta} \xi\right)^{\alpha}-1}-\frac{e^{i \theta}}{-i \xi-e^{i \theta}}+\frac{e^{-i \theta}}{-i \xi+e^{-i \theta}}\right), \quad \operatorname{Im} \xi \geqslant 0 .
$$


Indeed: the pole of $\alpha /\left(\left(-i e^{-i \theta} \xi\right)^{\alpha}-1\right)$ is cancelled by $e^{i \theta} /\left(-i \xi-e^{i \theta}\right)$. Furthermore, the estimate $|\Phi(\xi)| \leqslant C \min \left\{1,|\xi|^{-1}\right\}$ holds in the upper complex half-plane, so that $\Phi$ is in the Hardy space $\mathscr{H}^{2}$ in the upper complex half-plane. Hence, by Theorem 2.16, for $\xi>0$ we have

$$
\mathscr{F} G(\xi)=\Phi(i \xi)=\frac{1}{2 \pi i} \int_{-\infty}^{\infty} \frac{\Phi(t)}{t-i \xi} d t, \quad 0=\frac{1}{2 \pi i} \int_{-\infty}^{\infty} \frac{\Phi(t)}{t+i \xi} d t .
$$

It follows that

$$
\begin{aligned}
\mathscr{F} G(\xi) & =\Phi(i \xi)+\overline{0}=\frac{1}{2 \pi i} \int_{-\infty}^{\infty} \frac{\Phi(t)-\overline{\Phi(t)}}{t-i \xi} d t \\
& =\frac{1}{\pi} \int_{-\infty}^{\infty} \frac{\operatorname{Im} \Phi(t)}{t-i \xi} d t=-\frac{1}{\pi} \int_{0}^{\infty} \frac{\operatorname{Im} \Phi(-t)}{t+i \xi} d t+\frac{1}{\pi} \int_{0}^{\infty} \frac{\operatorname{Im} \Phi(t)}{t-i \xi} d t
\end{aligned}
$$

(here we added the sides of the first equality in (3.2) and complex conjugates of the corresponding sides of the other equality in (3.2)). By a straightforward calculation, for $t>0$ we have

$$
\begin{aligned}
\operatorname{Im} \Phi(t) & =\sin \frac{\pi}{\alpha} \operatorname{Im}\left(\frac{\alpha}{e^{-i \alpha(\pi / 2+\theta)} t^{\alpha}-1}-\frac{1}{e^{-i(\pi / 2+\theta)} t-1}-\frac{1}{e^{i(\pi / 2+\theta)} t-1}\right) \\
& =\sin \frac{\pi}{\alpha} \times \frac{\alpha t^{\alpha} \sin \left(\alpha\left(\frac{\pi}{2}+\theta\right)\right)}{t^{2 \alpha}-2 t^{\alpha} \cos \left(\alpha\left(\frac{\pi}{2}+\theta\right)\right)+1} .
\end{aligned}
$$

Similarly, for $t>0$,

$$
\operatorname{Im} \Phi(-t)=-\sin \frac{\pi}{\alpha} \times \frac{\alpha t^{\alpha} \sin \left(\alpha\left(\frac{\pi}{2}-\theta\right)\right)}{t^{2 \alpha}-2 t^{\alpha} \cos \left(\alpha\left(\frac{\pi}{2}-\theta\right)\right)+1} .
$$

In particular, $\operatorname{Im} \Phi(t)$ is integrable over $t \in \mathbb{R}$.

Observe that for $t>0$, the functions $\xi \mapsto 1 /(t-i \xi)$ and $\xi \mapsto 1 /(t+i \xi)$ are Fourier transforms of $x \mapsto e^{t x} \chi_{(-\infty, 0)}(x)$ and $x \mapsto e^{-t x} \chi_{(0, \infty)}(x)$, respectively. By Fubini's theorem, (1.1) and (3.3), the Fourier transform of the function

$$
G^{+}(x)=-\frac{1}{\pi} \int_{0}^{\infty} \operatorname{Im} \Phi(-t) e^{-t x} \chi_{(0, \infty)}(x) d t+\frac{1}{\pi} \int_{0}^{\infty} \operatorname{Im} \Phi(t) e^{t x} \chi_{(-\infty, 0)}(x) d t
$$

coincides with $\mathscr{F} G$ on $(0, \infty)$. Since both $G^{+}$and $G$ are real-valued, we conclude that $\mathscr{F} G^{+}=\mathscr{F} G$ on $\mathbb{R}$, and consequently $G^{+}$and $G$ are equal almost everywhere.

Finally, for $x>0$ the functions $G^{+}(x)=G^{-}(-x)$ and $G^{+}(-x)=G^{-}(x)$ are Laplace transforms of nonnegative functions (see (1.1)), and hence they are completely monotone.

We will later see that

$$
G^{+}\left(0^{+}\right)=\sin \left(\theta+\frac{\pi}{\alpha}-\frac{\pi}{2}\right), \quad G^{+}\left(0^{-}\right)=\sin \left(-\theta+\frac{\pi}{\alpha}-\frac{\pi}{2}\right) .
$$

In particular,

$$
G^{+}\left(0^{+}\right)-G^{+}\left(0^{-}\right)=2 \sin \frac{\pi}{\alpha} \sin \theta .
$$

We will need the following regularity result.

Lemma 3.1. The function $G^{+}$is Hölder continuous with exponent $\alpha-1$, save for a jump at $x=0$. More precisely, the function

$$
g(x)=G^{+}(x) \chi_{\mathbb{R} \backslash\{0\}}(x)-G^{+}\left(0^{+}\right) \chi_{(0, \infty)}-G^{+}\left(0^{-}\right) \chi_{(-\infty, 0)}
$$

is Hölder continuous: there exists a constant $C$ such that

$$
|g(x)-g(y)| \leqslant C|x-y|^{\alpha-1}, \quad x, y \in \mathbb{R} .
$$


Proof. We consider first an auxiliary function $f(x)=G^{+}(x)-c e^{-x} \chi_{(0, \infty)}(x)$, where

$$
c=2 \sin \frac{\pi}{\alpha} \sin \theta=-i \sin \frac{\pi}{\alpha}\left(e^{i \theta}-e^{-i \theta}\right) .
$$

Note that $f$ is continuous on $\mathbb{R} \backslash\{0\}$, and

$$
\begin{aligned}
\mathscr{F} f(\xi) & =\sin \frac{\pi}{\alpha}\left(\frac{\alpha}{\psi(\xi)-1}-\frac{e^{i \theta}}{\xi-e^{i \theta}}+\frac{e^{-i \theta}}{\xi+e^{-i \theta}}\right)+\frac{c i}{\xi-i} \\
& =\sin \frac{\pi}{\alpha}\left(\frac{\alpha}{\psi(\xi)-1}-e^{i \theta}\left(\alpha \frac{1}{\xi-e^{i \theta}}-\frac{1}{\xi-i}\right)+e^{-i \theta}\left(\frac{1}{\xi+e^{-i \theta}}-\frac{1}{\xi-i}\right)\right) .
\end{aligned}
$$

It follows that $\mathscr{F} f(\xi)=O\left(|\xi|^{-\alpha}\right)+O\left(|\xi|^{-2}\right)=O\left(|\xi|^{-\alpha}\right)$ as $|\xi| \rightarrow \infty$, and therefore $|\mathscr{F} f(\xi)| \leqslant C_{1} /\left(1+|\xi|^{\alpha}\right)$ for $\xi \in \mathbb{R}$. In particular, $\mathscr{F} f$ is integrable, and hence $f$, modified appropriately at 0 , is a continuous function. Furthermore, for $x, y \in \mathbb{R}$,

$$
\begin{aligned}
|f(x)-f(y)| & =\left|\int_{-\infty}^{\infty}\left(e^{i \xi x}-e^{i \xi y}\right) \mathscr{F} f(\xi) d \xi\right| \leqslant \int_{-\infty}^{\infty} \min \{2,|x-y||\xi|\} \frac{C_{1}}{|\xi|^{\alpha}} d \xi \\
& =|x-y|^{\alpha-1} \int_{-\infty}^{\infty} \min \{2,|t|\} \frac{C_{1}}{|t|^{\alpha}} d t=C_{2}|x-y|^{\alpha-1}
\end{aligned}
$$

we used a substitution $\xi=|x-y|^{-1} t$ in the penultimate step. Thus, $f$ is Hölder continuous with exponent $\alpha-1$.

It remains to observe that $g-f$ (modified appropriately at zero) is bounded and Lipschitz continuous. Indeed, by definition, for some constants $c_{1}, c_{2}, c_{3}, c_{4}$ we have $g(x)-f(x)=\left(c_{1}+c_{2} e^{-x}\right) \chi_{(0, \infty)}(x)+\left(c_{3}+c_{4} e^{x}\right) \chi_{(-\infty, 0)}(x)$ when $x \neq 0$. Since both $f$ and $g$ are continuous at zero, we necessarily have $c_{1}+c_{2}=c_{3}+c_{4}$, and consequently $g-f$ is Lipschitz continuous.

\section{Spectral expansion of transition operators $P_{t}^{0}$}

In this section we estabilish a generalised eigenfunction expansion for the transition operators $P_{t}^{0}$.

\subsection{Multiplication by test functions}

Recall that the Laplace transform of the transition density $p_{t}^{0}(x, y)$ with respect to $t$ is equal to the potential kernel $u_{\lambda}^{0}(x, y)$. Our first goal is to apply (2.2) to express the Laplace transform of $\int_{-\infty}^{\infty} \int_{-\infty}^{\infty} f(x) g(y) p_{t}^{0}(x, y) d x d y$ with respect to $t$ in terms of Fourier transforms of $f$ and $g$, where $f$ and $g$ are suitable test functions.

Lemma 4.1. If $f$ and $g$ are in both $\mathscr{L}^{1}(\mathbb{R})$ and $\mathscr{L}^{2}(\mathbb{R})$, then

$$
\begin{aligned}
\int_{0}^{\infty} & \int_{-\infty}^{\infty} \int_{-\infty}^{\infty} e^{-\lambda t} f(x) g(y) p_{t}^{0}(x, y) d x d y d t \\
= & \frac{1}{2 \pi} \int_{-\infty}^{\infty} \frac{\mathscr{F} f(-\xi) \mathscr{F} g(\xi)}{\lambda+\psi(\xi)} d \xi \\
& \quad-\frac{1}{u_{\lambda}(0)}\left(\frac{1}{2 \pi} \int_{-\infty}^{\infty} \frac{\mathscr{F} f(-\xi)}{\lambda+\psi(\xi)} d \xi\right)\left(\frac{1}{2 \pi} \int_{-\infty}^{\infty} \frac{\mathscr{F} g(\eta)}{\lambda+\psi(\eta)} d \eta\right)
\end{aligned}
$$

for all $\lambda>0$.

Proof. Recall that $p_{t}^{0}(x, y) \geqslant 0$ and $\int_{0}^{\infty} e^{-\lambda t} p_{t}^{0}(x, y) d t=u_{\lambda}^{0}(x, y) \leqslant u_{\lambda}(y-x) \leqslant u_{\lambda}(0)$. Thus, if $f$ and $g$ are integrable functions, then $e^{-\lambda t} f(x) g(y) p_{t}^{0}(x, y)$ is integrable with 
respect to $t>0$ and $x, y \in \mathbb{R}$. By Fubini's theorem and (2.2),

$$
\begin{aligned}
\int_{0}^{\infty} & \int_{-\infty}^{\infty} \int_{-\infty}^{\infty} e^{-\lambda t} f(x) g(y) p_{t}^{0}(x, y) d x d y d t \\
& =\int_{-\infty}^{\infty} \int_{-\infty}^{\infty} f(x) g(y) u_{t}^{0}(x, y) d x d y \\
& =\int_{-\infty}^{\infty} \int_{-\infty}^{\infty} f(x) g(y)\left(u_{\lambda}(y-x)-\frac{u_{\lambda}(-x) u_{\lambda}(y)}{u_{\lambda}(0)}\right) d x d y .
\end{aligned}
$$

Suppose additionally that $f, g \in \mathscr{L}^{2}(\mathbb{R})$. Since $\mathscr{F} u_{\lambda}(\xi)=1 /(\lambda+\psi(-\xi))$ is in $\mathscr{L}^{2}(\mathbb{R})$, we have $u_{\lambda} \in \mathscr{L}^{2}(\mathbb{R})$ and, by Plancherel's theorem,

$$
\begin{aligned}
\int_{-\infty}^{\infty} f(x) u_{\lambda}(y-x) d x & =\frac{1}{2 \pi} \int_{-\infty}^{\infty} e^{i \xi y} \mathscr{F} f(\xi) \mathscr{F} u_{\lambda}(\xi) d \xi, \\
\int_{-\infty}^{\infty} g(y) u_{\lambda}(y) d y & =\frac{1}{2 \pi} \int_{-\infty}^{\infty} \mathscr{F} g(\eta) \mathscr{F} u_{\lambda}(-\eta) d \eta .
\end{aligned}
$$

It follows that

$$
\begin{aligned}
\int_{0}^{\infty} & \int_{-\infty}^{\infty} \int_{-\infty}^{\infty} e^{-\lambda t} f(x) g(y) p_{t}^{0}(x, y) d x d y d t \\
= & \frac{1}{2 \pi} \int_{-\infty}^{\infty}\left(\int_{-\infty}^{\infty} \mathscr{F} f(\xi) e^{i \xi y} g(y) \mathscr{F} u_{\lambda}(\xi) d \xi\right) d y \\
& \quad-\frac{1}{u_{\lambda}(0)}\left(\frac{1}{2 \pi} \int_{-\infty}^{\infty} \mathscr{F} f(\xi) \mathscr{F} u_{\lambda}(\xi) d \xi\right)\left(\frac{1}{2 \pi} \int_{-\infty}^{\infty} \mathscr{F} g(\eta) \mathscr{F} u_{\lambda}(-\eta) d \eta\right) .
\end{aligned}
$$

Since $\mathscr{F} f(\xi) \mathscr{F} u_{\lambda}(\xi)$ and $g(y)$ are integrable, once again applying Fubini's theorem, we eventually find that

$$
\begin{aligned}
\int_{0}^{\infty} & \int_{-\infty}^{\infty} \int_{-\infty}^{\infty} e^{-\lambda t} f(x) g(y) p_{t}^{0}(x, y) d x d y d t \\
= & \frac{1}{2 \pi} \int_{-\infty}^{\infty} \mathscr{F} f(\xi) \mathscr{F} g(-\xi) \mathscr{F} u_{\lambda}(\xi) d \xi \\
& \quad-\frac{1}{u_{\lambda}(0)}\left(\frac{1}{2 \pi} \int_{-\infty}^{\infty} \mathscr{F} f(\xi) \mathscr{F} u_{\lambda}(\xi) d \xi\right)\left(\frac{1}{2 \pi} \int_{-\infty}^{\infty} \mathscr{F} g(\eta) \mathscr{F} u_{\lambda}(-\eta) d \eta\right) .
\end{aligned}
$$

The desired result follows from $\mathscr{F} u_{\lambda}(\xi)=1 /(\lambda+\psi(-\xi))$ after substituting $\xi$ for $-\xi$.

In [23], the process $X_{t}$ is assumed to be symmetric, and so $\psi$ is real-valued. In this case inversion of the Laplace transform in $t$ in (4.1) is possible by extending analytically the right-hand side to $\lambda \in \mathbb{C} \backslash(-\infty, 0]$, and writing down a Stieltjes-like representation in terms of boundary values along $(-\infty, 0)$. In the non-symmetric case this approach is problematic: the right-hand side of (4.1) no longer automatically extends to an analytic function in $\mathbb{C} \backslash(-\infty, 0]$. A way around if found by considering more regular test functions, and deforming the contour of integration in $\xi$ and $\eta$ so that $\psi(\xi)$ and $\psi(\eta)$ are again real-valued.

\subsection{Contour deformation}

Throughout this section, we fix $f, g \in \mathcal{H}$. Recall that $f$ and $g$ are real-valued, and their Laplace transforms are entire functions such that $\mathscr{L} f(\bar{\xi})=\overline{\mathscr{L} f(\xi)}, \mathscr{L} g(\bar{\xi})=\overline{\mathscr{L} g(\xi)}$. By Lemma 2.3, $|\mathscr{L} f(\xi)|$ and $|\mathscr{L} g(\xi)|$ are bounded by $C \min \left\{1,|\xi|^{-1}\right\}$ in every closed sector which contains neither $(0, \infty)$ nor $(-\infty, 0)$. 
We will constantly use the following notation. For $r>0$ we let

$$
\begin{aligned}
& h_{0}(r)=\cos \theta \\
& h_{1}(r)=\operatorname{Re}\left(e^{i \theta} \mathscr{L} f\left(-i r e^{i \theta}\right)\right), \\
& h_{2}(r)=\operatorname{Re}\left(e^{i \theta} \mathscr{L} g\left(i r e^{i \theta}\right)\right), \\
& h_{3}(r)=\operatorname{Re}\left(e^{i \theta} \mathscr{L} f\left(-i r e^{i \theta}\right) \mathscr{L} g\left(i r e^{i \theta}\right)\right) .
\end{aligned}
$$

For $j=0,1,2,3$ we define

$$
\phi_{j}(\lambda)=\frac{1}{\pi} \int_{0}^{\infty} \frac{h_{j}(r)}{r^{\alpha}+\lambda} d r, \quad \lambda \in \mathbb{C} \backslash(-\infty, 0] .
$$

Note that

$$
\phi_{0}(\lambda)=\frac{\cos \theta}{\pi} \frac{1}{\lambda^{1-1 / \alpha}} \int_{0}^{\infty} \frac{1}{t^{\alpha}+1} d t=\frac{\cos \theta}{\alpha \sin \frac{\pi}{\alpha}} \frac{1}{\lambda^{1-1 / \alpha}} \int_{0}^{\infty} \frac{1}{t^{\alpha}+1} d t .
$$

Observe also that if $f(x)=g(-x)$, then $h_{2}(r)=h_{1}(r)$. Finally, we set

$$
\phi_{4}(\lambda)=\phi_{3}(\lambda)-\frac{\phi_{1}(\lambda) \phi_{2}(\lambda)}{\phi_{0}(\lambda)}, \quad \lambda \in \mathbb{C} \backslash(-\infty, 0],
$$

and for later needs we extend the above definitions to $(-\infty, 0)$ by the formula

$$
\phi_{j}(-\lambda):=\lim _{\varepsilon \rightarrow 0^{+}} \phi_{j}(-\lambda+i \varepsilon), \quad \lambda>0 .
$$

The following result is a variant of Lemma 4.1 after appropriate contour deformation.

Lemma 4.2. With the above assumptions and notation, we have

$$
\int_{0}^{\infty} \int_{-\infty}^{\infty} \int_{-\infty}^{\infty} e^{-\lambda t} f(x) g(y) p_{t}^{0}(x, y) d x d y d t=\phi_{4}(\lambda), \quad \lambda>0 .
$$

Proof. To simplify the notation, we consider only $\theta \geqslant 0$. The remaining case $\theta<0$ is completely analogous. Fix $\lambda>0$ and denote the left-hand side of (4.2) by $I$. By Lemma 4.1,

$$
\begin{aligned}
I= & \frac{1}{2 \pi} \int_{-\infty}^{\infty} \frac{\mathscr{L} f(-i \xi) \mathscr{L} g(i \xi)}{\lambda+\psi(\xi)} d \xi \\
& \quad-\frac{1}{u_{\lambda}(0)}\left(\frac{1}{2 \pi} \int_{-\infty}^{\infty} \frac{\mathscr{L} f(-i \xi)}{\lambda+\psi(\xi)} d \xi\right)\left(\frac{1}{2 \pi} \int_{-\infty}^{\infty} \frac{\mathscr{L} g(i \eta)}{\lambda+\psi(\eta)} d \eta\right) .
\end{aligned}
$$

Now we deform the contour of integration $\mathbb{R}$ to $\left(-e^{-i \theta} \infty, 0\right) \cup\left(0, e^{i \theta} \infty\right)$ in each of the three integrals in the right-hand side.

Recall that $\psi\left(e^{i \theta} r\right)=\psi\left(-e^{-i \theta} r\right)=r^{\alpha}$. Observe that if $|\operatorname{Arg} \xi| \leqslant|\theta|$, then

$$
\begin{aligned}
|\operatorname{Arg} \psi(\xi)| & =\left|\operatorname{Arg}\left(e^{-i \theta} \xi\right)^{\alpha}\right|=|-\alpha \theta+\alpha \operatorname{Arg} \xi| \leqslant 2 \alpha \theta \\
& \leqslant 2 \alpha\left(\frac{\pi}{\alpha}-\frac{\pi}{2}\right)=(2-\alpha) \pi,
\end{aligned}
$$

and hence

$$
\begin{aligned}
|\lambda+\psi(\xi)|^{2} & =\lambda^{2}+|\psi(\xi)|^{2}+2 \lambda \operatorname{Re} \psi(\xi) \geqslant \lambda^{2}+|\psi(\xi)|^{2}+2 \lambda|\psi(\xi)| \cos ((2-\alpha) \pi) \\
& \geqslant c(\alpha)(\lambda+|\psi(\xi)|)^{2} \geqslant c(\alpha, \lambda)(1+|\psi(\xi)|)^{2} .
\end{aligned}
$$




\section{Spectral theory for stable processes}

Since $|\psi(\xi)|=|\xi|^{\alpha}$, we conclude that

$$
|\lambda+\psi(\xi)| \geqslant c(\alpha, \lambda)\left(1+|\xi|^{\alpha}\right) .
$$

Now we use Lemma 2.18: the function $\mathscr{L} f(-i \xi) \mathscr{L} g(i \xi) /(\lambda+\psi(\xi))$ is analytic in $\{\xi \in \mathbb{C}$ : $\operatorname{Re} \xi>0\}$, and by Lemma 2.3 and (4.6) we have:

$$
\begin{gathered}
|\mathscr{L} f(-i \xi)| \leqslant C \min \left(1,|\xi|^{-1}\right), \quad|\mathscr{L} g(i \xi)| \leqslant C \min \left(1,|\xi|^{-1}\right), \\
\frac{1}{|\lambda+\psi(\xi)|} \leqslant C(\alpha, \lambda) \min \left\{1,|\xi|^{-\alpha}\right\}
\end{gathered}
$$

in the sector $\{\xi \in \mathbb{C}: 0 \leqslant \arg (\xi) \leqslant \theta\}$. Therefore, by Lemma 2.3 ),

$$
\begin{aligned}
\frac{1}{2 \pi} \int_{(0, \infty)} \frac{\mathscr{L} f(-i \xi) \mathscr{L} g(i \xi)}{\lambda+\psi(\xi)} d \xi & =\frac{1}{2 \pi} \int_{\left(0, e^{i \theta} \infty\right)} \frac{\mathscr{L} f(-i \xi) \mathscr{L} g(i \xi)}{\lambda+\psi(\xi)} d \xi \\
& =\frac{1}{2 \pi} \int_{0}^{\infty} \frac{e^{i \theta} \mathscr{L} f\left(-i e^{i \theta} r\right) \mathscr{L} g\left(i e^{i \theta} r\right)}{\lambda+r^{\alpha}} d r .
\end{aligned}
$$

In a similar way,

$$
\begin{aligned}
\frac{1}{2 \pi} \int_{(-\infty, 0)} \frac{\mathscr{L} f(-i \xi) \mathscr{L} g(i \xi)}{\lambda+\psi(\xi)} d \xi & =\frac{1}{2 \pi} \int_{\left(-e^{-i \theta} \infty, 0\right)} \frac{\mathscr{L} f(-i \xi) \mathscr{L} g(i \xi)}{\lambda+\psi(\xi)} d \xi \\
& =\frac{1}{2 \pi} \int_{0}^{\infty} \frac{e^{-i \theta} \mathscr{L} f\left(i e^{-i \theta} r\right) \mathscr{L} g\left(-i e^{-i \theta} r\right)}{\lambda+r^{\alpha}} d r .
\end{aligned}
$$

Now, we combine the above two identities: since

$$
e^{i \theta} \mathscr{L} f\left(-i e^{i \theta} r\right) \mathscr{L} g\left(i e^{i \theta} r\right)=\overline{e^{-i \theta} \mathscr{L} f\left(i e^{-i \theta} r\right) \mathscr{L} g\left(-i e^{-i \theta} r\right)},
$$

we find that

$$
\begin{aligned}
& \frac{1}{2 \pi} \int_{-\infty}^{\infty} \frac{\mathscr{L} f(-i \xi) \mathscr{L} g(i \xi)}{\lambda+\psi(\xi)} d \xi \\
& \quad=\frac{1}{2 \pi} \int_{(0, \infty)} \frac{\mathscr{L} f(-i \xi) \mathscr{L} g(i \xi)}{\lambda+\psi(\xi)} d \xi+\frac{1}{2 \pi} \int_{(-\infty, 0)} \frac{\mathscr{L} f(-i \xi) \mathscr{L} g(i \xi)}{\lambda+\psi(\xi)} d \xi \\
& \quad=\frac{1}{2 \pi} \int_{0}^{\infty} \frac{e^{-i \theta} \mathscr{L} f\left(i e^{-i \theta} r\right) \mathscr{L} g\left(-i e^{-i \theta} r\right)+e^{i \theta} \mathscr{L} f\left(-i e^{i \theta} r\right) \mathscr{L} g\left(i e^{i \theta} r\right)}{\lambda+r^{\alpha}} d r \\
& \quad=\frac{1}{\pi} \int_{0}^{\infty} \frac{\operatorname{Re}\left(e^{-i \theta} \mathscr{L} f\left(i e^{-i \theta} r\right) \mathscr{L} g\left(-i e^{-i \theta} r\right)\right)}{\lambda+r^{\alpha}} d r=\phi_{3}(\lambda) .
\end{aligned}
$$

The same argument applies to the other two integrals in the right-hand side of (4.3), which are found to be equal to $\phi_{1}(\lambda)$ and $\phi_{2}(\lambda)$, respectively. Finally, again by the same argument,

$$
u_{\lambda}(0)=\frac{1}{2 \pi} \int_{-\infty}^{\infty} \frac{1}{\lambda+\psi(-\eta)} d \eta=\frac{1}{2 \pi} \int_{0}^{\infty} \frac{e^{i \theta}+e^{-i \theta}}{\lambda+r^{\alpha}} d r=\phi_{0}(\lambda) .
$$

We conclude that

$$
I=\phi_{3}(\lambda)-\frac{\phi_{1}(\lambda) \phi_{2}(\lambda)}{\phi_{0}(\lambda)}=\phi_{4}(\lambda), \quad \lambda>0,
$$

and the proof is complete. 


\subsection{Application of the Cauchy's integral formula}

We consider $f, g \in \mathcal{H}$, and we continue to use the notation introduced in the previous section. We now use Cauchy's integral formula given in Theorem 2.16 for the function $\sqrt{\xi} \phi_{4}(\xi)$. The following set of lemmas justify the application of this result. First we check that $\phi_{4} \in \mathscr{N}^{+}$.

Lemma 4.3. The function $\lambda \phi_{4}\left(-\lambda^{2}\right)$ is in the Nevanlinna class $\mathscr{N}^{+}$.

Proof. Recall that for $j=0,1,2,3$ and $\lambda \in \mathbb{C} \backslash(-\infty, 0]$, we have

$$
\phi_{j}(\lambda)=\frac{1}{\pi} \int_{0}^{\infty} \frac{h_{j}(r)}{r^{\alpha}+\lambda} d r=\frac{1}{\alpha \pi} \int_{0}^{\infty} \frac{s^{1 / \alpha-1} h_{j}\left(s^{1 / \alpha}\right)}{s+\lambda} d s .
$$

Therefore, if $\operatorname{Im} \lambda>0$, then

$$
\begin{aligned}
\lambda \phi_{j}\left(-\lambda^{2}\right) & =\frac{1}{\alpha \pi} \int_{0}^{\infty} \frac{\lambda s^{1 / \alpha-1} h_{j}\left(s^{1 / \alpha}\right)}{s-\lambda^{2}} d s \\
& =\frac{1}{2 \alpha \pi} \int_{0}^{\infty} \frac{s^{1 / \alpha-1} h_{j}\left(s^{1 / \alpha}\right)}{s-\lambda} d s-\frac{1}{2 \alpha \pi} \int_{0}^{\infty} \frac{s^{1 / \alpha-1} h_{j}\left(s^{1 / \alpha}\right)}{s+\lambda} d s .
\end{aligned}
$$

Writing $h_{j}(r)=\max \left\{h_{j}(r), 0\right\}-\max \left\{-h_{j}(r), 0\right\}$, we see that $\lambda \phi_{j}\left(-\lambda^{2} \lambda\right)=\phi_{j, 1}(-\lambda)-$ $\phi_{j, 2}(-\lambda)-\phi_{j, 3}(\lambda)+\phi_{j, 4}(\lambda)$ for appropriate Stieltjes functions $\phi_{j, 1}, \phi_{j, 2}, \phi_{j, 3}, \phi_{j, 4}$. By Lemmas 2.12 and 2.13, $\lambda \phi_{j}\left(-\lambda^{2}\right)$ is in the Nevanlinna class $\mathscr{N}^{+}$.

Similarly, we show that $\lambda \phi_{0}\left(-\lambda^{2}\right)$ is an outer function. We have $h_{0}\left(s^{1 / \alpha}\right) \geqslant 0$ in (4.9), and hence $\operatorname{Im}\left(\lambda \phi_{0}\left(-\lambda^{2}\right)\right) \geqslant 0$ whenever $\operatorname{Im} \lambda>0$. By Lemma 2.12, $-i \lambda \phi_{0}\left(-\lambda^{2}\right)$ is an outer function, and hence also $\lambda \phi_{0}\left(-\lambda^{2}\right)$ is an outer function.

Lemma 2.13 implies now that the function $\phi_{4}=\phi_{3}-\phi_{1} \phi_{2} / \phi_{0}$ is in the Nevanlinna class $\mathscr{N}^{+}$.

Next, we study the boundary values of $\phi_{4}$. This will involve the following pricipal value integrals:

$$
K_{j}(s)=\frac{1}{\pi} \text { p.v. } \int_{0}^{\infty} \frac{h_{j}(r) d r}{r^{\alpha}-s^{\alpha}}, \quad s>0,
$$

where $j=0,1,2,3$. Note that since $h_{0}(r)=\cos \theta$, we have

$$
K_{0}(s)=-\frac{\cot \frac{\pi}{\alpha} \cos \theta}{\alpha s^{\alpha-1}}, \quad s>0 ;
$$

see, for example, [23], Example 5.1. For $j=0,1,2,3$, define also

$$
L_{j}(s)=\frac{h_{j}(s)}{\alpha s^{\alpha-1}}, \quad s>0 .
$$

In particular,

$$
L_{0}(s)=\frac{\cos \theta}{\alpha s^{\alpha-1}}, \quad s>0 .
$$

Lemma 4.4. With the above notation, $\phi_{4}$ extends to a continuous function in the closed upper complex half-plane, except possibly at 0 . If this extension is denoted by the same symbol $\phi_{4}$, then we have

$$
\begin{aligned}
\operatorname{Im} \phi_{4}\left(-s^{\alpha}\right) & =-L_{3}(s)+\frac{L_{1}(s) L_{2}(s)}{L_{0}(s)} \\
& -\left(\operatorname{Im} \frac{1}{K_{0}(s)-i L_{0}(s)}\right)^{-1} \operatorname{Im} \frac{K_{1}(s)-i L_{1}(s)}{K_{0}(s)-i L_{0}(s)} \operatorname{Im} \frac{K_{2}(s)-i L_{2}(s)}{K_{0}(s)-i L_{0}(s)} .
\end{aligned}
$$


Proof. Since for $f \in \mathcal{H}$ the function $\mathscr{L} f$ is an entire function, the functions $h_{j}, j=$ $0,1,2,3$, are continuously differentiable on $(0, \infty)$. Thus, $\phi_{j}, j=0,1,2,3$, extend to continuous functions in the upper complex half-plane, except possibly at 0 ; we denote these extensions again by $\phi_{j}$. Now, by Sokhozki's formula (note that $\frac{h_{j}\left(t^{1 / \alpha}\right)}{\alpha t^{1-1 / \alpha}}$ are also continuously differentiable near $t=s^{\alpha}$ ), for $j=0,1,2,3$ and $s>0$ we have

$$
\begin{aligned}
\phi_{j}\left(-s^{\alpha}\right) & =\lim _{\varepsilon \rightarrow 0^{+}} \phi_{j}\left(-s^{\alpha}+i \varepsilon\right)=\lim _{\varepsilon \rightarrow 0^{+}} \frac{1}{\pi} \int_{0}^{\infty} \frac{h_{j}(r)}{r^{\alpha}-s^{\alpha}+i \varepsilon} d r \\
& =\lim _{\varepsilon \rightarrow 0^{+}} \frac{1}{\pi} \int_{0}^{\infty} \frac{h_{j}\left(t^{\frac{1}{\alpha}}\right)}{t-s^{\alpha}+i \varepsilon} \frac{d t}{\alpha t^{1-\frac{1}{\alpha}}}= \\
& =\lim _{\varepsilon \rightarrow 0^{+}} \frac{1}{\pi} \int_{0}^{\infty} \frac{t-s^{\alpha}}{\left(t-s^{\alpha}\right)^{2}+\varepsilon^{2}} \frac{h_{j}\left(t^{\frac{1}{\alpha}}\right)}{\alpha t^{1-\frac{1}{\alpha}}} d t-\lim _{\varepsilon \rightarrow 0^{+}} \frac{1}{\pi} \int_{0}^{\infty} \frac{i \varepsilon}{\left(t-s^{\alpha}\right)^{2}+\varepsilon^{2}} \frac{h_{j}\left(t^{\frac{1}{\alpha}}\right)}{\alpha t^{1-\frac{1}{\alpha}}} d t \\
& =\text { p.v. } \int_{0}^{\infty} \frac{1}{t-s^{\alpha}} \frac{h_{j}\left(t^{\frac{1}{\alpha}}\right)}{\alpha t^{1-\frac{1}{\alpha}}} d t-\frac{h_{j}\left(\left(s^{\alpha}\right)^{\frac{1}{\alpha}}\right)}{\alpha\left(s^{\alpha}\right)^{1-\frac{1}{\alpha}}} \\
& =\frac{1}{\pi} \text { p.v. } \int_{0}^{\infty} \frac{h_{j}(r) d r}{r^{\alpha}-s^{\alpha}}-\frac{i}{\alpha s^{\alpha-1}} \frac{\pi h_{j}(s)}{\pi}=K_{j}(s)-i L_{j}(s) ;
\end{aligned}
$$

see [44], Section 1.8, or a similar calculation in the proof of Lemma 4.3 and Section 2.1 in [23]. Hence, we can express $\phi_{4}\left(-s^{\alpha}\right)$ as

$$
\phi_{4}\left(-s^{\alpha}\right)=K_{3}(s)-i L_{3}(s)-\frac{\left(K_{1}(s)-i L_{1}(s)\right)\left(K_{2}(s)-i L_{2}(s)\right)}{K_{0}(s)-i L_{0}(s)} .
$$

Now, since $K_{j}$ and $L_{j}$ are real-valued for $j=0,1,2,3$, the desired result follows by Lemma 2.20 .

The following estimates will be used to prove square-integrability of $s \phi_{4}\left(-s^{2}\right)$ for the application of Theorem 2.16.

Lemma 4.5. With the above notation and assumptions, there is a constant $C$ such that for $j=1,2,3$ we have

$$
\left|K_{j}(s)\right| \leqslant \begin{cases}C s^{1-\alpha} & \text { if } 0<s<1, \\ C s^{-\alpha} \log (1+s) & \text { if } s \geqslant 1\end{cases}
$$

Proof. By Lemmas 2.3 and 2.4, the functions $h_{1}$ and $h_{2}$ satisfy the assumptions of Lemma 2.19, and consequently

$$
\left|K_{j}(s)\right| \leqslant \begin{cases}C s^{1-\alpha} & \text { if } 0<s<1, \\ C s^{-\alpha} \log (1+s) & \text { if } s \geqslant 1\end{cases}
$$

for $j=1,2$. Similarly, again by Lemmas 2.3 and 2.4 , we have $\left|h_{3}(r)\right| \leqslant C \min \left\{1, r^{-2}\right\}$ and $\left|h_{3}^{\prime}(r)\right| \leqslant C \min \left\{1, r^{-3}\right\}$, so Lemma 2.19 applies also $h_{3}$, leading to the desired bound for $K_{3}$.

Lemma 4.6. With the above notation and assumptions, there is a constant $C$ such that

$$
\left|\phi_{4}(-\lambda)\right| \leqslant \begin{cases}C \lambda^{1 / \alpha-1} & \text { if } 0<\lambda<1 \\ C \lambda^{-1}(\log (1+\lambda))^{2} & \text { if } \lambda \geqslant 1\end{cases}
$$

Proof. For $s>0$, we have

$$
\begin{aligned}
\left|\phi_{4}\left(-s^{\alpha}\right)\right| & =\left|\phi_{3}\left(-s^{\alpha}\right)-\frac{\phi_{1}\left(-s^{\alpha}\right) \phi_{2}\left(-s^{\alpha}\right)}{\phi_{0}\left(-s^{\alpha}\right)}\right| \\
& \leqslant\left|K_{3}(s)\right|+\left|L_{3}(s)\right|+\frac{\left(\left|K_{1}(s)\right|+\left|L_{1}(s)\right|\right)\left(\left|K_{2}(s)\right|+\left|L_{2}(s)\right|\right)}{\left|L_{0}(s)\right|} .
\end{aligned}
$$


Recall that $L_{0}(s)=\cos \theta /\left(\alpha s^{\alpha-1}\right)$ for $s>0$. By definition and Lemma 2.3, there is a constant $C_{1}$ such that if $s>0$, then $\left|L_{j}(s)\right| \leqslant C_{1} s^{1-\alpha} \min \left\{1, s^{-1}\right\}$ for $j=1,2$, and $\left|L_{3}(s)\right| \leqslant$ $C_{1} s^{1-\alpha} \min \left\{1, s^{-2}\right\}$. Similar estimates for $K_{j}(s), j=1,2,3$, are given in Lemma 4.5. It follows that for some constant $C_{2}$ we have

$$
\left|\phi_{4}\left(-s^{\alpha}\right)\right| \leqslant C_{2} s^{1-\alpha}, \quad 0<s<1,
$$

and

$$
\left|\phi_{4}\left(-s^{\alpha}\right)\right| \leqslant C_{2} s^{-\alpha}(\log (1+s))^{2}, \quad s \geqslant 1
$$

as desired.

We are now ready to apply Theorem 2.16.

Theorem 4.7. Let $f, g \in \mathcal{H}$ and $t>0$. Then, with the above notation,

$$
\int_{-\infty}^{\infty} \int_{-\infty}^{\infty} f(x) g(y) p_{t}^{0}(x, y) d x d y=-\frac{1}{\pi} \int_{0}^{\infty} e^{-r t} \operatorname{Im} \phi_{4}(-r) d r
$$

where $\operatorname{Im} \phi_{4}$ is given by Lemma 4.4.

Proof. Recall that by Lemma 4.2,

$$
\int_{0}^{\infty} \int_{-\infty}^{\infty} \int_{-\infty}^{\infty} e^{-\lambda t} f(x) g(y) p_{t}^{0}(x, y) d x d y d t=\phi_{4}(\lambda), \quad \lambda>0 .
$$

We verify that the function $\sqrt{\lambda} \phi_{4}(\lambda)$ satisfies the assumptions of Corollary 2.17. By Lemma 4.3, the function $\lambda \phi_{4}\left(\lambda^{2}\right)$ is in the Nevanlinna class $\mathscr{N}^{+}$. By Lemma 4.4, this function extends continuously to the closed upper complex half-plane, except possibly at $\lambda=0$, and, by Lemma 4.6, this extension satisfies

$$
\left|\sqrt{\lambda} \phi_{4}(-\lambda)\right| \leqslant \begin{cases}C \lambda^{1 / \alpha-1 / 2} & \text { if } 0<\lambda<1 \\ C \lambda^{-1 / 2}(\log (1+\lambda))^{2} & \text { if } \lambda \geqslant 1 .\end{cases}
$$

In particular,

$$
\int_{0}^{\infty}\left|\lambda \phi_{4}\left(-\lambda^{2}\right)\right|^{2} d \lambda \leqslant C^{2} \int_{0}^{1} \lambda^{2 / \alpha-1} d \lambda+C^{2} \int_{1}^{\infty} \lambda^{-2}\left(\log \left(1+\lambda^{2}\right)\right)^{4} d \lambda<\infty .
$$

Therefore, the assumptions of Corollary 2.17 are satisfied.

We conclude that for $\lambda \in \mathbb{C} \backslash(-\infty, 0]$,

$$
\sqrt{\lambda} \phi_{4}(\lambda)=\frac{1}{\pi} \int_{0}^{\infty} \frac{\sqrt{\lambda}}{\sqrt{r}} \frac{\operatorname{Re}\left(i \sqrt{r} \phi_{4}(-r)\right)}{r+\lambda} d r=-\frac{\sqrt{\lambda}}{\pi} \int_{0}^{\infty} \frac{\operatorname{Im} \phi_{4}(-r)}{r+\lambda} d r,
$$

which implies that for $\lambda>0$,

$$
\int_{0}^{\infty} \int_{-\infty}^{\infty} \int_{-\infty}^{\infty} e^{-\lambda t} f(x) g(y) p_{t}^{0}(x, y) d x d y d t=-\frac{1}{\pi} \int_{0}^{\infty} \int_{0}^{\infty} \operatorname{Im} \phi_{4}(-r) e^{-r t} e^{-\lambda r} d t d r .
$$

The desired result for almost every $t>0$ follows by Fubini's theorem and uniqueness of Laplace transforms. Extension to all $t>0$ is a consequence of continuity. Indeed, the right-hand side of (4.14) is clearly continuous in $t>0$. Continuity of the left-hand side results from integrability of $f(x) g(y)$ with respect to $x, y \in \mathbb{R}$, and continuity of $t \mapsto p_{t}^{0}(\cdot, \cdot)$ on $(0, \infty)$ with respect to the topology of uniform convergence. 


\subsection{Generalised eigenfunction expansion of $P_{t}^{0}$}

Our goal in this section is to express $\operatorname{Im} \phi_{4}(-\lambda)$ (see Lemma 4.4) in terms of $f$ and $g$ rather than the Laplace transforms of $f$ and $g$. This result, combined with Theorem 4.7, will prove Theorem 1.2. We use the notation introduced in Sections 4.2 and 4.3.

The expression for $\operatorname{Im} \phi_{4}(-\lambda)$ in Lemma 4.4:

$$
\begin{aligned}
\operatorname{Im} \phi_{4}\left(-s^{\alpha}\right) & =-L_{3}(s)+\frac{L_{1}(s) L_{2}(s)}{L_{0}(s)} \\
& -\left(\operatorname{Im} \frac{1}{K_{0}(s)-i L_{0}(s)}\right)^{-1} \operatorname{Im} \frac{K_{1}(s)-i L_{1}(s)}{K_{0}(s)-i L_{0}(s)} \operatorname{Im} \frac{K_{2}(s)-i L_{2}(s)}{K_{0}(s)-i L_{0}(s)},
\end{aligned}
$$

has two parts. The former one is expanded in Lemma 4.8; the latter one is more involved and it is studied in Lemma 4.11, after a number of auxiliary results.

Lemma 4.8. With the above assumptions and notation,

$$
\begin{aligned}
L_{3}(s) & -\frac{L_{1}(s) L_{2}(s)}{L_{0}(s)} \\
= & \frac{1}{\alpha s^{\alpha-1} \cos \theta}\left(\int_{-\infty}^{\infty} e^{-s x \sin \theta} \sin (s x \cos \theta) f(x) d x\right)\left(\int_{-\infty}^{\infty} e^{s y \sin \theta} \sin (s y \cos \theta) g(y) d y\right) .
\end{aligned}
$$

Proof. Recall that for $j=0,1,2,3, L_{j}(s)=h_{j}(s) /\left(\alpha s^{\alpha-1}\right)$, where

$$
\begin{array}{llrl}
h_{0}(s) & =\cos \theta, & h_{1}(s) & =\operatorname{Re}\left(e^{i \theta} \mathscr{L} f\left(-i s e^{i \theta}\right)\right), \\
h_{2}(s) & =\operatorname{Re}\left(e^{i \theta} \mathscr{L} g\left(i s e^{i \theta}\right)\right), & h_{3}(s) & =\operatorname{Re}\left(e^{i \theta} \mathscr{L} f\left(-i s e^{i \theta}\right) \mathscr{L} g\left(i s e^{i \theta}\right)\right) .
\end{array}
$$

For a fixed $s>0$, denote $\operatorname{Re} \mathscr{L} f\left(-i s e^{i \theta}\right)=a_{1}, \operatorname{Im} \mathscr{L} f\left(-i s e^{i \theta}\right)=b_{1}, \operatorname{Re} \mathscr{L} g\left(i s e^{i \theta}\right)=a_{2}$, $\operatorname{Im} \mathscr{L} g\left(i s e^{i \theta}\right)=b_{2}$. We have

$$
\begin{aligned}
L_{1}(s) & =\frac{\operatorname{Re}\left(e^{i \theta} \mathscr{L} f\left(-i s e^{i \theta}\right)\right)}{\alpha s^{\alpha-1}}=\frac{a_{1} \cos \theta-b_{1} \sin \theta}{\alpha s^{\alpha-1}}, \\
L_{2}(s) & =\frac{\operatorname{Re}\left(e^{i \theta} \mathscr{L} g\left(i s e^{i \theta}\right)\right)}{\alpha s^{\alpha-1}}=\frac{a_{2} \cos \theta-b_{2} \sin \theta}{\alpha s^{\alpha-1}}, \\
L_{3}(s) & =\frac{\operatorname{Re}\left(e^{i \theta} \mathscr{L} f\left(-i s e^{i \theta}\right) \mathscr{L} g\left(i s e^{i \theta}\right)\right)}{\alpha s^{\alpha-1}}=\frac{\left(a_{1} a_{2}-b_{1} b_{2}\right) \cos \theta-\left(a_{1} b_{2}+a_{2} b_{1}\right) \sin \theta}{\alpha s^{\alpha-1}} .
\end{aligned}
$$

It follows that

$$
\begin{aligned}
L_{3}(r)-\frac{L_{1}(r) L_{2}(r)}{L_{0}(r)}= & \frac{\left(a_{1} a_{2}-b_{1} b_{2}\right) \cos \theta-\left(a_{1} b_{2}+a_{2} b_{1}\right) \sin \theta}{\alpha s^{\alpha-1}} \\
& -\frac{\left(a_{1} \cos \theta-b_{1} \sin \theta\right)\left(a_{2} \cos \theta-b_{2} \sin \theta\right)}{\alpha s^{\alpha-1} \cos \theta} \\
= & -\frac{b_{1} b_{2} \cos \theta}{\alpha s^{\alpha-1}}-\frac{\left.b_{1} b_{2} \sin ^{2} \theta\right)}{\alpha s^{\alpha-1} \cos \theta}=-\frac{b_{1} b_{2}}{\alpha s^{\alpha-1} \cos \theta} .
\end{aligned}
$$

Since

$$
\begin{aligned}
& b_{1}=\operatorname{Im} \mathscr{L} f\left(-i s e^{i \theta}\right)=\int_{-\infty}^{\infty} e^{-s x \sin \theta} \sin (s x \cos \theta) f(x) d x, \\
& b_{2}=\operatorname{Im} \mathscr{L} g\left(i s e^{i \theta}\right)=-\int_{-\infty}^{\infty} e^{s y \sin \theta} \sin (s y \cos \theta) g(y) d y
\end{aligned}
$$

the proof is complete.

Recall that every $f \in \mathcal{H}$ can be written as $f=f_{+}+f_{-}$, where $f_{+}=f \chi_{(0, \infty)} \in \mathcal{H}_{+}$and $f_{-}=f \chi_{(-\infty, 0)} \in \mathcal{H}_{-}$. For the next result, we need the following variant of Sokhozki's formula. 
Lemma 4.9. If $s>0, \zeta=s e^{i \theta}$ or $\zeta=-s e^{-i \theta}, f \in \mathcal{H}$ and $f=f_{+}+f_{-}$as above, then

$$
\frac{1}{2 \pi i} \text { p.v. } \int_{\left(-e^{-i \theta} \infty, 0\right) \cup\left(0, e^{i \theta} \infty\right)} \frac{\mathscr{L} f(-i \xi)}{\xi-\zeta} d \xi=\frac{1}{2} \mathscr{L} f_{+}(-i \zeta)-\frac{1}{2} \mathscr{L} f_{-}(-i \zeta) .
$$

Here $\left(-e^{-i \theta} \infty, 0\right) \cup\left(0, e^{i \theta} \infty\right)$ denotes a contour that consists of two rays, $\operatorname{Arg} \xi=\theta$ and $\operatorname{Arg}(-\xi)=-\theta$, oriented in such a way that the real part increases along this contour.

Proof. Let $\Gamma_{R}$ denote the boundary of the circular sector

$$
D_{R}=\left\{\xi \in \mathbb{C}:|\xi| \leqslant R,|\arg (-i \xi)| \leqslant \frac{\pi}{2}-\theta\right\},
$$

oriented in a counter-clockwise manner. Since $\left|\mathscr{L} f_{+}(-i \xi)\right| \leqslant C \min \left\{1,|\xi|^{-1}\right\}$ in $D_{R}$ by Lemma 2.3, we have

$$
\frac{1}{2 \pi i} \text { p.v. } \int_{\left(-e^{-i \theta} \infty, 0\right) \cup\left(0, e^{i \theta} \infty\right)} \frac{\mathscr{L} f_{+}(-i \xi)}{\xi-\zeta} d \xi=\lim _{R \rightarrow \infty} \frac{1}{2 \pi i} \text { p.v. } \int_{\Gamma_{R}} \frac{\mathscr{L} f_{+}(-i \xi)}{\xi-\zeta} d \xi .
$$

If $R>s$, then $\mathscr{L} f_{+}(-i \xi)$ is analytic in the neighbourhood of $D_{R}$ and $\zeta \in \Gamma_{R}$. Thus, by the usual Sokhozki's formula, the expression under the limit in the above equality is equal to $\frac{1}{2} \mathscr{L} f_{+}(-i \zeta)$.

A similar argument applies to $f_{-}$rather than $f_{+}$, but here we need to consider the boundary $\Gamma_{R}$ of the circular sector

$$
D_{R}=\left\{\xi \in \mathbb{C}:|\xi| \leqslant R,|\arg (i \xi)| \leqslant \frac{\pi}{2}+\theta\right\},
$$

oriented in a clockwise manner. Note that $\left|\mathscr{L} f_{-}(-i \xi)\right| \leqslant C \min \left\{1,|\xi|^{-1}\right\}$ in $D_{R}$, and so we have a complete analogue of (4.16) for $f_{-}$. However, the expression under the limit in the right-hand side is now equal to $-\frac{1}{2} \mathscr{L} f_{-}(-i \zeta)$ (with a minus sign) due to clockwise orientation of $\Gamma_{R}$. The assertion of the lemma follows by combining the above results for $f_{+}$and $f_{-}$.

Observe that for $f \in \mathcal{H}$ and $r>0$,

$$
e^{i \theta} \mathscr{L} f\left(-i s e^{i \theta}\right)=\int_{-\infty}^{\infty} e^{-s x \sin \theta}(\cos (s x \cos \theta+\theta)+i \sin (s x \cos \theta+\theta)) f(x) d x .
$$

Lemma 4.10. With the above assumptions and notation, for $s>0$ we have

$$
\begin{aligned}
& K_{1}(s)=\frac{1}{\alpha s^{\alpha-1}} \int_{-\infty}^{\infty}\left(\frac{G^{+}(s x)}{\sin \frac{\pi}{\alpha}}-e^{-s x \sin \theta} \sin (s x \cos \theta+\theta) \operatorname{sign} x\right) f(x) d x, \\
& K_{2}(s)=\frac{1}{\alpha s^{\alpha-1}} \int_{-\infty}^{\infty}\left(\frac{G^{+}(-s x)}{\sin \frac{\pi}{\alpha}}-e^{s x \sin \theta} \sin (s x \cos \theta-\theta) \operatorname{sign} x\right) g(x) d x .
\end{aligned}
$$

Proof. Fix $s>0$. By definition,

$$
\begin{aligned}
K_{1}(s) & =\frac{1}{\pi} \text { p.v. } \int_{0}^{\infty} \frac{\operatorname{Re}\left(e^{i \theta} \mathscr{L} f\left(-i r e^{i \theta}\right)\right)}{r^{\alpha}-s^{\alpha}} d r \\
& =\frac{1}{2 \pi} \text { p.v. } \int_{0}^{\infty} \frac{e^{i \theta} \mathscr{L} f\left(-i r e^{i \theta}\right)}{r^{\alpha}-s^{\alpha}} d r+\frac{1}{2 \pi} \text { p.v. } \int_{0}^{\infty} \frac{e^{-i \theta} \mathscr{L} f\left(i r e^{-i \theta}\right)}{r^{\alpha}-s^{\alpha}} d r \\
& =\frac{1}{2 \pi} \text { p.v. } \int_{\left(-e^{-i \theta} \infty, 0\right) \cup\left(0, e^{i \theta} \infty\right)} \frac{\mathscr{L} f(-i \xi)}{\psi(\xi)-s^{\alpha}} d \xi .
\end{aligned}
$$


The function $1 /\left(\psi(\xi)-s^{\alpha}\right)$ is meromorphic in $\mathbb{C} \backslash i \mathbb{R}$, with two simple poles at $\xi=s e^{i \theta}$ and $\xi=-s e^{-i \theta}$. The corresponding residues are $1 / \psi^{\prime}\left(s e^{i \theta}\right)=e^{i \theta} /\left(\alpha s^{\alpha-1}\right)$ and $1 / \psi^{\prime}\left(-s e^{-i \theta}\right)=$ $-e^{-i \theta} /\left(\alpha s^{\alpha-1}\right)$, respectively. Removing this poles leads to the identity

$$
\begin{aligned}
K_{1}(s) & =\frac{1}{2 \pi} \int_{\left(-e^{-i \theta} \infty, 0\right) \cup\left(0, e^{i \theta} \infty\right)} \Phi(\xi) \mathscr{L} f(-i \xi) d \xi \\
& +\frac{1}{2 \pi} \text { p.v. } \int_{\left(-e^{-i \theta} \infty, 0\right) \cup\left(0, e^{i \theta} \infty\right)}\left(\frac{e^{i \theta}}{\alpha s^{\alpha-1}} \frac{1}{\xi-s e^{i \theta}}-\frac{e^{-i \theta}}{\alpha s^{\alpha-1}} \frac{1}{\xi+s e^{-i \theta}}\right) \mathscr{L} f(-i \xi) d \xi,
\end{aligned}
$$

where

$$
\Phi(\xi)=\frac{1}{\psi(\xi)-s^{\alpha}}-\frac{e^{i \theta}}{\alpha s^{\alpha-1}} \frac{1}{\xi-s e^{i \theta}}+\frac{e^{-i \theta}}{\alpha s^{\alpha-1}} \frac{1}{\xi+s e^{-i \theta}}, \quad \xi \in \mathbb{C} \backslash i \mathbb{R} .
$$

The second term in the above expression for $K_{1}(s)$ is given by Lemma 4.9: it is equal to

$$
\begin{aligned}
& \frac{i e^{i \theta}}{2 \alpha s^{\alpha-1}}\left(\mathscr{L} f_{+}\left(-i s e^{i \theta}\right)-\mathscr{L} f_{-}\left(-i s e^{i \theta}\right)\right)-\frac{i e^{-i \theta}}{2 \alpha s^{\alpha-1}}\left(\mathscr{L} f_{+}\left(i s e^{-i \theta}\right)-\mathscr{L} f_{-}\left(i s e^{-i \theta}\right)\right) \\
& \quad=\frac{1}{\alpha s^{\alpha-1}}\left(\operatorname{Im}\left(e^{i \theta} \mathscr{L} f_{-}\left(-i s e^{i \theta}\right)\right)-\operatorname{Im}\left(e^{i \theta} \mathscr{L} f_{+}\left(-i s e^{i \theta}\right)\right)\right) \\
& \quad=\frac{1}{\alpha s^{\alpha-1}}\left(\int_{-\infty}^{0} e^{s x \sin \theta} \sin (s x \cos \theta+\theta) f(x) d x-\int_{0}^{\infty} e^{s x \sin \theta} \sin (s x \cos \theta+\theta) f(x) d x\right)
\end{aligned}
$$

(in the last step we used the fact that $f_{+}=f \chi_{(0, \infty)}$ and $\left.f_{-}=f \chi_{(-\infty, 0)}\right)$. To identify the first term in the right-hand side of (4.18), recall that the function $G^{+}$was defined so that

$$
\mathscr{F} G^{+}(\xi)=\sin \frac{\pi}{\alpha}\left(\frac{\alpha}{\psi(\xi)-1}-\frac{1}{e^{-i \theta} \xi-1}+\frac{1}{e^{i \theta} \xi+1}\right), \quad \xi \in \mathbb{R} .
$$

Therefore, for $s>0$, the Fourier transform of $G_{s}^{+}(x)=G^{+}(s x)$ is given by

$$
\begin{aligned}
\mathscr{F} G_{s}^{\uparrow}(\xi) & =\frac{\sin \frac{\pi}{\alpha}}{s}\left(\frac{\alpha}{\psi(\xi / s)-1}-\frac{1}{e^{-i \theta} \xi / s-1}+\frac{1}{e^{i \theta} \xi / s+1}\right) \\
& =s^{\alpha-1} \sin \frac{\pi}{\alpha}\left(\frac{\alpha}{\psi(\xi)-s^{\alpha}}-\frac{e^{i \theta}}{s^{\alpha-1}} \frac{1}{\xi-s e^{i \theta}}+\frac{e^{i \theta}}{s^{\alpha-1}} \frac{1}{\xi+s e^{-i \theta}}\right)
\end{aligned}
$$

for $\xi \in \mathbb{R}$. It follows that $\Phi(\xi)=\mathscr{F} G_{s}^{\uparrow}(\xi) /\left(\alpha s^{\alpha-1} \sin \frac{\pi}{\alpha}\right)$ for $\xi \in \mathbb{R}$. Recall that $\Phi(\xi)$ is an analytic function in $\mathbb{C} \backslash i \mathbb{R}$, bounded by $C \min \left\{1,|\xi|^{-1}\right\}$ (see the proof of Proposition 1.3). Similarly, $\mathscr{L} f(-i \xi)$ is an analytic function in $\mathbb{C} \backslash i \mathbb{R}$, bounded by $C \min \left\{1,|\xi|^{-1}\right\}$ in the sector $\{\xi \in \mathbb{C}:|\arg \xi| \leqslant|\theta|\}$ (by Lemma 2.3). It follows that we can deform the contour of integration in the first term of the right-hand side of (4.18) to $\mathbb{R}$, which leads to the integral

$$
\begin{aligned}
\frac{1}{2 \pi} \int_{-\infty}^{\infty} \Phi(\xi) \mathscr{L} f(-i \xi) d \xi & =\frac{1}{2 \pi \alpha s^{\alpha-1} \sin \frac{\pi}{\alpha}} \int_{-\infty}^{\infty} \mathscr{F} f(-\xi) \mathscr{F} G_{s}^{\uparrow}(\xi) d \xi \\
& =\frac{1}{\alpha s^{\alpha-1} \sin \frac{\pi}{\alpha}} \int_{-\infty}^{\infty} f(x) G_{s}^{\uparrow}(x) d x ;
\end{aligned}
$$

we used Plancherel's theorem in the last step. The desired expression for $K_{1}(s)$ follows. The expression for $K_{2}(s)$ is obtained from that for $K_{1}(s)$ by considering $f(x)=g(-x)$.

Lemma 4.11. With the above assumptions and notation, for $s>0$ we have

$$
\operatorname{Im} \frac{1}{K_{0}(s)-i L_{0}(s)}=\frac{\alpha s^{\alpha-1} \sin ^{2} \frac{\pi}{\alpha}}{\cos \theta}
$$




$$
\begin{aligned}
& \operatorname{Im} \frac{K_{1}(s)-i L_{1}(s)}{K_{0}(s)-i L_{0}(s)}=\frac{\sin \frac{\pi}{\alpha}}{\cos \theta} \int_{-\infty}^{\infty}\left(G^{+}(s x)+e^{-s x \sin \theta} \cos \left(s x \cos \theta+\theta+\frac{\pi}{\alpha} \operatorname{sign} x\right)\right) f(x) d x, \\
& \operatorname{Im} \frac{K_{2}(s)-i L_{2}(s)}{K_{0}(s)-i L_{0}(s)}=\frac{\sin \frac{\pi}{\alpha}}{\cos \theta} \int_{-\infty}^{\infty}\left(G^{+}(-s y)+e^{s y \sin \theta} \cos \left(s y \cos \theta-\theta+\frac{\pi}{\alpha} \operatorname{sign} y\right)\right) g(y) d y .
\end{aligned}
$$

Proof. We fix $s>0$. For simplicity, in this proof we write $K_{j}$ and $L_{j}$ rather than $K_{j}(s)$ and $L_{j}(s)$. Recall that $K_{0}=-\cot \frac{\pi}{\alpha} \cos \theta /\left(\alpha s^{\alpha-1}\right)$ and $L_{0}=\cos \theta /\left(\alpha s^{\alpha-1}\right)$. Thus,

$$
K_{0}^{2}+L_{0}^{2}=\left(\frac{\cos \theta}{\alpha s^{\alpha-1} \sin \frac{\pi}{\alpha}}\right)^{2}, \quad \operatorname{Im} \frac{1}{K_{0}-i L_{0}}=\frac{L_{0}}{K_{0}^{2}+L_{0}^{2}}=\frac{\alpha s^{\alpha-1} \sin ^{2} \frac{\pi}{\alpha}}{\cos \theta}
$$

formula (4.19a) follows. Furthermore,

$$
\operatorname{Im} \frac{K_{1}-i L_{1}}{K_{0}-i L_{0}}=\frac{K_{1} L_{0}-K_{0} L_{1}}{K_{0}^{2}+L_{0}^{2}}=\frac{\alpha s^{\alpha-1} \sin \frac{\pi}{\alpha}}{\cos \theta}\left(K_{1} \sin \frac{\pi}{\alpha}+L_{1} \cos \frac{\pi}{\alpha}\right)
$$

The expressions for $K_{1}$ is given in Lemma 4.10 , while $L_{1}$ is given by

$$
L_{1}=\frac{\operatorname{Re}\left(e^{i \theta} \mathscr{L} f\left(-i s e^{i \theta}\right)\right)}{\alpha s^{\alpha-1}}=\frac{1}{\alpha s^{\alpha-1}} \int_{-\infty}^{\infty} e^{-s x \sin \theta} \cos (s x \cos \theta+\theta) f(x) d x .
$$

It follows that

$$
\begin{aligned}
& \operatorname{Im} \frac{K_{1}-i L_{1}}{K_{0}-i L_{0}}=\frac{\sin \frac{\pi}{\alpha}}{\cos \theta} \int_{-\infty}^{\infty}\left(G^{+}(s x)-e^{-s x \sin \theta} \sin (s x \cos \theta+\theta) \sin \frac{\pi}{\alpha} \operatorname{sign} x\right. \\
&\left.+e^{-s x \sin \theta} \cos (s x \cos \theta+\theta) \cos \frac{\pi}{\alpha}\right) f(x) d x .
\end{aligned}
$$

This proves (4.19b). Formula (4.19c) follows from (4.19b) by substituting $f(x)=g(-x)$.

By combining the above lemmas, we can finally prove Theorem 1.2.

Proof of the Theorem 1.2. Fix $t>0$. By Theorem 4.7 and substitution $r=s^{\alpha}$, we have

$$
\begin{aligned}
\int_{-\infty}^{\infty} \int_{-\infty}^{\infty} f(x) g(y) p_{t}^{0}(x, y) d x d y & =-\frac{1}{2 \pi} \int_{0}^{\infty} e^{-r t} \operatorname{Im} \phi_{4}(-r) d r \\
& =-\frac{1}{2 \pi} \int_{0}^{\infty} e^{-s^{\alpha} t} \alpha s^{\alpha-1} \operatorname{Im} \phi_{4}\left(-s^{\alpha}\right) d s
\end{aligned}
$$

Lemma 4.4 provides an expression for $\operatorname{Im} \phi_{4}\left(-s^{\alpha}\right)$. Combining it with Lemmas 4.8 and 4.11 , we obtain

$$
\begin{aligned}
\int_{-\infty}^{\infty} \int_{-\infty}^{\infty} f(x) g(y) p_{t}^{0}(x, y) d x d y \\
=\int_{0}^{\infty} \frac{e^{-s^{\alpha} t}}{\cos \theta}\left(\int_{-\infty}^{\infty} e^{-s x \sin \theta} \sin (s x \cos \theta) f(x) d x\right)\left(\int_{-\infty}^{\infty} e^{s y \sin \theta} \sin (s y \cos \theta) g(y) d y\right) d s \\
+\int_{0}^{\infty} \frac{e^{-s^{\alpha} t}}{\cos \theta}\left(\int_{-\infty}^{\infty}\left(G^{+}(s x)+e^{-s x \sin \theta} \cos \left(s x \cos \theta+\theta+\frac{\pi}{\alpha} \operatorname{sign} x\right)\right) f(x) d x\right) \times \\
\quad \times\left(\int_{-\infty}^{\infty}\left(G^{+}(-s y)+e^{s y \sin \theta} \cos \left(s y \cos \theta-\theta+\frac{\pi}{\alpha} \operatorname{sign} y\right)\right) g(y) d y\right) d s .
\end{aligned}
$$


Thus, the last two integrands in the right-hand side are equal to $-F^{+}(s x) f(x)$ and $-F^{+}(-s y) g(y)$, respectively. Indeed, for $s>0$ we have

$$
\begin{aligned}
F^{+}(s x) & =e^{-s x \sin \theta} \sin \left(s|x| \cos \theta+\theta \operatorname{sign} x+\frac{\pi}{\alpha}-\frac{\pi}{2}\right)-G^{+}(s x) \\
& =-e^{-s x \sin \theta} \cos \left(s|x| \cos \theta+\theta \operatorname{sign} x+\frac{\pi}{\alpha}\right)-G^{+}(s x) \\
& =-e^{-s x \sin \theta} \cos \left(s x \cos \theta+\theta+\frac{\pi}{\alpha} \operatorname{sign} x\right)-G^{+}(s x),
\end{aligned}
$$

and a similar formula holds for $F^{+}(-s y)$. The proof is complete.

\section{Hitting time}

In this section we obtain the formula for $P\left(\tau^{0}>t\right)$. Our starting point is the expression for the Laplace transform of $\tau^{0}$ :

$$
\int_{0}^{\infty} e^{-\lambda t} \mathbb{P}^{x}\left(\tau_{0}>t\right) d t=\frac{1-\mathbb{E}^{x} e^{-\lambda \tau_{0}}}{\lambda}=\frac{1}{\lambda}-\frac{u_{\lambda}(-x)}{\lambda u_{\lambda}(0)}
$$

(see (2.4)). Our plan is as follows: with the notation of Section 4.2, we define

$$
\phi_{5}(\lambda)=\frac{\mathscr{L} g(0)}{\lambda}-\frac{\phi_{2}(\lambda)}{\lambda \phi_{0}(\lambda)}
$$

when $\lambda \in \mathbb{C} \backslash(-\infty, 0]$, and, as usual, we let $\phi_{5}(-\lambda)=\lim _{\varepsilon \rightarrow 0^{+}} \phi_{5}(-\lambda+i \varepsilon)$ when $\lambda>0$. First, we will show that whenever $g \in \mathcal{H}$, we have

$$
\begin{aligned}
& \int_{0}^{\infty} \int_{\mathbb{R}} e^{-\lambda t} \mathbb{P}^{x}\left(\tau_{0}>t\right) g(x) d x d t=-\frac{1}{\pi} \int_{0}^{\infty} \int_{0}^{\infty} e^{-\lambda t} e^{-r t} \operatorname{Im} \phi_{5}(-r) d r d t \\
&=-\frac{1}{\pi \cos \theta} \int_{0}^{\infty} \int_{0}^{\infty} e^{-\lambda t} e^{-s^{\alpha} t} \int_{\mathbb{R}} \frac{F^{-}(s x)}{s} g(x) d x d s d t .
\end{aligned}
$$

Then, we will change the order of integration. The desired result will then follow by a density-type argument: the class of admissible functions $g$ is dense in an appropriate sense, and both sides of (1.2) are continuous functions of $x$. Note, however, that changing the order of integration is not merely an application of Fubini's theorem: the integral in the right-hand side of (5.1) is not absolutely convergent. For this reason, we will first deform the contour of integration, only then apply Fubini's theorem, and then deform the contour back.

Lemma 5.1. The function $\lambda \phi_{5}\left(-\lambda^{2}\right)$ is in the Nevanlinna class $\mathcal{N}^{+}$, and it is in $\mathscr{L}^{p}(\mathbb{R})$ for some $p>1$.

Proof. The proof of the first part of the lemma is a minor modification of the proof of Lemma 4.3: the functions $\lambda$ and $\lambda \phi_{0}\left(-\lambda^{2}\right)$ are outer functions, $\lambda \phi_{2}\left(-\lambda^{2}\right)$ is in $\mathcal{N}^{+}$, and therefore $\lambda \phi_{5}\left(-\lambda^{2}\right)$ is in $\mathcal{N}^{+}$. In order to prove the other part of the lemma, we need to show that

$$
\int_{\mathbb{R}}\left|\lambda \phi_{5}\left(-\lambda^{2}\right)\right|^{2} d \lambda<\infty
$$

By Sokhozki formula (see the proof of Lemma 4.4), we have

$$
\begin{aligned}
\phi_{5}\left(-s^{\alpha}\right) & =\frac{\mathscr{L} g(0)}{-s^{\alpha}}-\frac{K_{2}(s)-i L_{2}(s)}{-s^{\alpha}\left(K_{0}(s)-i L_{0}(s)\right)} \\
& =\frac{K_{2}(s)-i L_{2}(s)-\left(K_{0}(s)-i L_{0}(s)\right) \mathscr{L} g(0)}{s^{\alpha}\left(K_{0}(s)-i L_{0}(s)\right)} .
\end{aligned}
$$

By the definition of $L_{2}(s)$, we have

$$
L_{2}(s)-L_{0}(s) \mathscr{L} g(0)=\frac{\operatorname{Re}\left(e^{i \theta} \mathscr{L} g\left(i s e^{i \theta}\right)\right)-\mathscr{L} g(0) \cos \theta}{\alpha s^{\alpha-1}}=\frac{\operatorname{Re}\left(e^{i \theta}\left(\mathscr{L} g\left(i s e^{i \theta}\right)-\mathscr{L} g(0)\right)\right)}{\alpha s^{\alpha-1}},
$$


and hence

$$
\left|L_{2}(s)-L_{0}(s) \mathscr{L} g(0)\right| \leqslant \frac{\left|\mathscr{L} g\left(i s e^{i \theta}\right)-\mathscr{L} g(0)\right|}{\alpha s^{\alpha-1}} .
$$

Using the bounds $|\mathscr{L} g(z)| \leqslant C \min \left\{1,|z|^{-1}\right\}$ and $\left|\mathscr{L} g^{\prime}(z)\right| \leqslant C \min \left\{1,|z|^{-2}\right\}$ (see Lemmas 2.3 and 2.4), we arrive at

$$
\left|L_{2}(s)-L_{0}(s) \mathscr{L} g(0)\right| \leqslant \frac{C \min \{s, 1\}}{\alpha s^{\alpha-1}} .
$$

In a very similar way, by the definition of $K_{2}(s)$,

$$
\begin{aligned}
K_{2}(s)-K_{0}(s) \mathscr{L} g(0) & =\frac{1}{\pi} \text { p.v. } \int_{0}^{\infty} \frac{\operatorname{Re}\left(e^{i \theta} \mathscr{L} g\left(i r e^{i \theta}\right)\right)-\mathscr{L} g(0) \cos \theta}{r^{\alpha}-s^{\alpha}} d r \\
& =\frac{1}{\pi} \text { p.v. } \int_{0}^{\infty} \frac{\operatorname{Re}\left(e^{i \theta}\left(\mathscr{L} g\left(i r e^{i \theta}\right)-\mathscr{L} g(0)\right)\right)}{r^{\alpha}-s^{\alpha}} d r,
\end{aligned}
$$

and by Lemmas 2.3 and 2.4, the function $h(r)=\mathscr{L} g\left(i r e^{i \theta}\right)-\mathscr{L} g(0)$ satisfies $|h(r)| \leqslant$ $C \min \{r, 1\}$ and $\left|h^{\prime}(r)\right| \leqslant C \min \left\{1, r^{-2}\right\}$. Repeating the proof of Lemma 2.19, with appropriately modified estimates (2.14), (2.15) and (2.16), we find that

$$
\left|K_{2}(s)-K_{0}(s) \mathscr{L} g(0)\right| \leqslant \frac{C_{1} \log (1+2 s)}{s^{\alpha-1}(1+s)}+C_{2} s^{1-\alpha} \min \{s, 1\}+C_{3} \min \left\{s^{1-\alpha}, 1\right\},
$$

and therefore

$$
\left|K_{2}(s)-K_{0}(s) \mathscr{L} g(0)\right| \leqslant C \min \left\{1, s^{1-\alpha}\right\} .
$$

The above bounds and the definitions of $K_{0}(s)$ and $L_{0}(s)$ imply that

$$
\left|\phi_{5}\left(-s^{\alpha}\right)\right| \leqslant \frac{C \min \left\{1, s^{1-\alpha}\right\}}{s^{\alpha} s^{1-\alpha}}=C \min \left\{s^{-1}, s^{-\alpha}\right\} .
$$

We conclude that

$$
\left|\lambda \phi_{5}\left(-\lambda^{2}\right)\right| \leqslant C \min \left\{\lambda^{1-2 / \alpha}, \lambda^{-1}\right\}
$$

so that $\lambda \phi_{5}\left(-\lambda^{2}\right)$ is in $\mathscr{L}^{p}(\mathbb{R})$ for every $p \in\left(1, \frac{\alpha}{2-\alpha}\right)$.

Lemma 5.2. We have, for $\lambda>0$,

$$
\phi_{5}(\lambda)=-\frac{1}{\pi} \int_{0}^{\infty} \int_{0}^{\infty} e^{-\lambda t} e^{-s t} \operatorname{Im} \phi_{5}(-s) d s d t .
$$

Proof. As in the proof of Theorem 4.7, we find that, by Lemma 5.1, the function $\sqrt{\lambda} \phi_{5}(\lambda)$ satisfies the assumptions of Corollary 2.17. It follows that for $\lambda \in \mathbb{C} \backslash(-\infty, 0]$,

$$
\sqrt{\lambda} \phi_{5}(\lambda)=-\frac{\sqrt{\lambda}}{\pi} \int_{0}^{\infty} \frac{\operatorname{Im} \phi_{5}(-r)}{r+\lambda} d r
$$

which implies (5.3).

Proof of Theorem 1.1. Let $g \in \mathcal{H}$. Recall that for $t>0$,

$$
\int_{0}^{\infty} e^{-\lambda t} \mathbb{P}^{x}\left(\tau_{0}>t\right) d t=\frac{1}{\lambda}-\frac{u_{\lambda}(-x)}{\lambda u_{\lambda}(0)} .
$$

Since $g$ is integrable, by Fubini's theorem, for every $\lambda>0$ we have

$$
\int_{0}^{\infty} \int_{\mathbb{R}} e^{-\lambda t} \mathbb{P}^{x}\left(\tau_{0}>t\right) g(x) d x d t=\frac{\mathscr{L} g(0)}{\lambda}-\frac{1}{\lambda u_{\lambda}(0)} \int_{\mathbb{R}} u_{\lambda}(-x) g(x) d x .
$$


By Plancherel's theorem and an argument used in Lemma 4.2,

$$
\int_{\mathbb{R}} u_{\lambda}(-x) g(x) d x=\frac{1}{2 \pi} \int_{\mathbb{R}} \frac{\mathscr{L} g(i \xi)}{\psi(\xi)+\lambda} d \xi=\phi_{2}(\lambda),
$$

and similarly $u_{\lambda}(0)=\phi_{0}(\lambda)$. Therefore,

$$
\int_{0}^{\infty} \int_{\mathbb{R}} e^{-\lambda t} \mathbb{P}^{x}\left(\tau_{0}>t\right) g(x) d x d t=\frac{\mathscr{L} g(0)}{\lambda}-\frac{\phi_{2}(\lambda)}{\lambda \phi_{0}(\lambda)}=\phi_{5}(\lambda) .
$$

By Lemma 5.2 we obtain

$$
\begin{aligned}
\int_{0}^{\infty} \int_{\mathbb{R}} e^{-\lambda t} \mathbb{P}^{x}\left(\tau_{0}>t\right) g(x) d x d t & =-\frac{1}{\pi} \int_{0}^{\infty} \int_{0}^{\infty} e^{-\lambda t} e^{-r t} \operatorname{Im} \phi_{5}(-r) d r d t \\
& =\frac{1}{\pi} \int_{0}^{\infty} \int_{0}^{\infty} e^{-\lambda t} e^{-s^{\alpha} t} \alpha s^{\alpha-1} \operatorname{Im} \frac{\phi_{2}\left(-s^{\alpha}\right)}{-s^{\alpha} \phi_{0}\left(-s^{\alpha}\right)} d s d t
\end{aligned}
$$

for every $\lambda>0$. From the uniqueness of the Laplace transform we get, for almost all $t>0$,

$$
\int_{\mathbb{R}} \mathbb{P}^{x}\left(\tau_{0}>t\right) g(x) d x=-\frac{\alpha}{\pi} \int_{0}^{\infty} \frac{e^{-s^{\alpha} t}}{s} \operatorname{Im} \frac{\phi_{2}\left(-s^{\alpha}\right)}{\phi_{0}\left(-s^{\alpha}\right)} d s .
$$

By Lemma (4.11), equality (4.20) and the fact that $\phi_{j}\left(-s^{\alpha}\right)=K_{j}(s)-i L_{j}(s), j=0,2$, we get

$$
\begin{aligned}
\operatorname{Im} \frac{\phi_{2}\left(-s^{\alpha}\right)}{\phi_{0}\left(-s^{\alpha}\right)} & =\operatorname{Im} \frac{K_{2}(s)-i L_{2}(s)}{K_{0}(s)-i L_{0}(s)} \\
& =\frac{\sin \frac{\pi}{\alpha}}{\cos \theta} \int_{-\infty}^{\infty}\left(G^{-}(s x)+e^{s x \sin \theta} \cos \left(s x \cos \theta-\theta+\frac{\pi}{\alpha} \operatorname{sign} x\right)\right) g(x) d x \\
& =-\frac{\sin \frac{\pi}{\alpha}}{\cos \theta} \int_{\mathbb{R}} F^{-}(s x) g(x) d x .
\end{aligned}
$$

We have thus proved that for almost all $t>0$,

$$
\int_{\mathbb{R}} \mathbb{P}^{x}\left(\tau_{0}>t\right) g(x) d x=\frac{\alpha \sin \frac{\pi}{\alpha}}{\pi \cos \theta} \int_{0}^{\infty} \frac{e^{-s^{\alpha} t}}{s} \int_{\mathbb{R}} F^{-}(s x) g(x) d x d s .
$$

We now change the order of integration in the right-hand side of (5.10). With no loss of generality, we assume that $\theta \geqslant 0$; the other case is dealt with in a similar manner.

Recall that

$$
F^{-}(s x)=e^{s x \sin \theta} \sin \left(s|x| \cos \theta-\theta \operatorname{sign} x+\frac{\pi}{\alpha}-\frac{\pi}{2}\right)-G^{-}(s x) .
$$

We split the integral in the right-hand side of (5.10) into three parts:

$$
\int_{0}^{\infty} e^{-s^{\alpha} t} \int_{\mathbb{R}} \frac{F^{-}(s x)}{s} g(x) d x d s=I_{1}+I_{2}+I_{3},
$$

where

$$
\begin{aligned}
& I_{1}=\int_{0}^{\infty} e^{-s^{\alpha} t} \int_{-\infty}^{0} \frac{F^{-}(s x)}{s} g(x) d x d s \\
& I_{2}=\int_{0}^{\infty} e^{-s^{\alpha} t} \int_{0}^{\infty} \frac{e^{-s x} \sin \varphi-G^{-}(s x)}{s} g(x) d x d s \\
& I_{3}=\int_{0}^{\infty} e^{-s^{\alpha} t} \int_{0}^{\infty} \frac{e^{s x \sin \theta} \sin (s|x| \cos \theta+\varphi)-e^{-s x} \sin \varphi}{s} g(x) d x d s,
\end{aligned}
$$

with the notation $\varphi=\frac{\pi}{\alpha}-\frac{\pi}{2}-\theta$. 
Since we assumed that $\theta \geqslant 0$, the function $e^{x \sin \theta} \sin (|x| \cos \theta+\varphi)$ is bounded and Lipschitz continuous on $(-\infty, 0]$. Recall that by Lemma 3.1, the function $G^{-}$is Hölder continuous on $(-\infty, 0)$ with exponent $\alpha-1$, and $G^{-}\left(0^{-}\right)=\sin \left(\frac{\pi}{\alpha}-\frac{\pi}{2}+\theta\right)=\sin \varphi$. It follows that $F^{-}$is Hölder continuous on $(-\infty, 0]$ with exponent $\alpha-1$, and $F^{-}(0)=0$. Furthermore, $F^{-}$is bounded on $(-\infty, 0]$, and hence $\left|F^{-}(s x)\right| \leqslant C \min \left\{1,(s|x|)^{\alpha-1}\right\}$ for $x \in(-\infty, 0]$. Finally, by the definition of $\mathcal{H}$, we have $|g(x)| \leqslant C \min \left\{1,|x|^{-\delta|x|}\right\}$. We conclude that the integrand in the double integral in the definition of $I_{1}$ is bounded by $C \min \left\{1,(s|x|)^{\alpha-1}\right\} s^{-1} \min \left\{1,|x|^{-\delta x}\right\} e^{-s^{\alpha} t}$, and

$$
\begin{aligned}
& \int_{-\infty}^{0} \int_{0}^{\infty} \min \left\{1,(s|x|)^{\alpha-1}\right\} s^{-1} \min \left\{1,|x|^{-\delta|x|}\right\} e^{-s^{\alpha} t} d s d x \\
& \quad \leqslant \int_{-\infty}^{0}\left(\int_{0}^{1 /|x|}(s|x|)^{\alpha-1} s^{-1} d s+\int_{1 /|x|}^{\infty} s^{-1} e^{-s^{\alpha} t} d s\right) \min \left\{1,|x|^{-\delta|x|}\right\} d x
\end{aligned}
$$

Clearly, $\int_{0}^{1 /|x|}(s|x|)^{\alpha-1} s^{-1} d s=|x|^{\alpha-1} \int_{0}^{1 /|x|} s^{\alpha-2} d s=\frac{1}{\alpha-1}$. Furthermore, if $|x|>1$, we have $\int_{1 /|x|}^{1} s^{-1} e^{-s^{\alpha} t} d s \leqslant \int_{1 /|x|}^{1} s^{-1} d s=\log |x| \leqslant \log (1+|x|)$. Finally, $\int_{1}^{\infty} s^{-1} e^{-s^{\alpha} t} d s \leqslant$ $\int_{1}^{\infty} e^{-s t} d s=\frac{1}{t} e^{-t} \leqslant \frac{1}{t}$. Hence,

$$
\begin{aligned}
& \int_{-\infty}^{0} \int_{0}^{\infty} \min \left\{1,(s|x|)^{\alpha-1}\right\} s^{-1} \min \left\{1,|x|^{-\delta|x|}\right\} e^{-s^{\alpha} t} d s d x \\
& \quad \leqslant \int_{-\infty}^{0}\left(\frac{1}{\alpha-1}+\log (1+|x|)+\frac{1}{t}\right) \min \left\{1,|x|^{-\delta|x|}\right\} d x<\infty .
\end{aligned}
$$

Thus, the integral in the definition $I_{1}$ converges absolutely, and by Fubini's theorem,

$$
I_{1}=\int_{-\infty}^{0} g(x) \int_{0}^{\infty} e^{-s^{\alpha} t} \frac{F^{-}(s x)}{s} d s d x .
$$

A similar argument applies to $I_{2}$ : again by Lemma 3.1, $e^{-s x} \sin \varphi-G^{-}(s x)$ is bounded and Hölder continuous on $(0, \infty)$ with right limit at 0 equal to zero, so that we may use Fubini's theorem. It follows that

$$
I_{2}=\int_{0}^{\infty} g(x) \int_{0}^{\infty} e^{-s^{\alpha} t} \frac{e^{-s x} \sin \varphi-G^{-}(s x)}{s} d s d x .
$$

The integral $I_{3}$, however, requires a more subtle treatment. We split it further into two parts, which are dealt with in a very similar way: since

$$
e^{s x \sin \theta} \sin (s x \cos \theta+\varphi)=\frac{e^{i\left(s x e^{-i \theta}+\varphi\right)}-e^{-i\left(s x e^{i \theta}+\varphi\right)}}{2 i},
$$

we have $I_{3}=\left(I_{4}-I_{5}\right) /(2 i)$, where

$$
\begin{aligned}
& I_{4}=e^{i \varphi} \int_{0}^{\infty} e^{-s^{\alpha} t} \int_{0}^{\infty} \frac{e^{i s x e^{-i \theta}}-e^{-s x}}{s} g(x) d x d s, \\
& I_{5}=e^{-i \varphi} \int_{0}^{\infty} e^{-s^{\alpha} t} \int_{0}^{\infty} \frac{e^{-i s x e^{i \theta}}-e^{-s x}}{s} g(x) d x d s .
\end{aligned}
$$

Recall that, by the definition of $\mathcal{H}, g$ extends to an analytic function in $\mathbb{C} \backslash i \mathbb{R}$, which is bounded by $C \min \left\{1,|x|^{-\delta|x|}\right\}$ in the sector $\{x \in \mathbb{C}:|\arg x| \leqslant|\theta|\}$. Furthermore, $\exp \left(i s x e^{-i \theta}\right)-\exp (-s x)$ is an entire function of $x$, bounded by $2 e^{s|x|}$. Hence we may deform the contour of integration in the inner integral from $(0, \infty)$ to $\left(0, e^{i \theta} \infty\right)$, and find 
that

$$
\begin{aligned}
I_{4} & =e^{i \varphi} \int_{0}^{\infty} e^{-s^{\alpha} t} \int_{\left(0, e^{i \theta} \infty\right)} \frac{e^{i s x e^{-i \theta}}-e^{-s x}}{s} g(x) d x d s \\
& =e^{i \varphi} \int_{0}^{\infty} e^{-s^{\alpha} t} \int_{0}^{\infty} \frac{e^{i r s}-e^{-r s e^{i \theta}}}{s} g\left(e^{i \theta} r\right) e^{i \theta} d r d s .
\end{aligned}
$$

The exponential function $z \mapsto e^{-z}$ is Lipschitz continuous in the right complex half-plane, with Lipschitz constant 1 . Therefore, $\left|e^{i r s+i \varphi}-e^{-r s e^{i \theta}+i \varphi}\right| \leqslant \min \{2,2 r s\}$ when $r, s>0$. By the argument used in the analysis of $I_{1}$, it follows that the double integral in (5.11) converges absolutely, and so, by Fubini's theorem,

$$
\begin{aligned}
I_{4} & =e^{i \varphi} \int_{0}^{\infty} g\left(e^{i \theta} r\right) e^{i \theta} \int_{0}^{\infty} e^{-s^{\alpha} t} \frac{e^{i r s}-e^{-r s e^{i \theta}}}{s} d s d r \\
& =e^{i \varphi} \int_{\left(0, e^{i \theta} \infty\right)} g(x) \int_{0}^{\infty} e^{-s^{\alpha} t} \frac{e^{i s x e^{-i \theta}}-e^{-s x}}{s} d s d x .
\end{aligned}
$$

We now deform the contour of integration with respect to $x$ from $\left(0, e^{i \theta} \infty\right)$ back to $(0, \infty)$. Note that the inner integral can be expressed by

$$
\begin{aligned}
& \int_{0}^{\infty} e^{-s^{\alpha}} t \frac{e^{i s x e^{-i \theta}}-e^{-s x}}{s} d s= \\
& =\int_{0}^{\infty} e^{-s^{\alpha} t} \frac{1-e^{-s x}}{s} d s-\int_{0}^{\infty} e^{-s^{\alpha} t} \frac{1-e^{i s x e^{-i \theta}}}{s} d s \\
& =\Phi(x)-\Phi\left(x e^{-i \theta}\right),
\end{aligned}
$$

where $\Phi(z)$ is the entire function defined in Lemma 2.21. Furthermore, if $0 \leqslant \operatorname{Arg} z \leqslant \theta$, then $-\theta \leqslant \operatorname{Arg}\left(z e^{-i \theta}\right) \leqslant 0$. Since $\theta<\frac{\pi}{2}<\frac{\pi}{2}+\frac{\pi}{2 \alpha}$, Lemma 2.21 implies that $\Phi(z)-\Phi\left(z e^{-i \theta}\right)$ is bounded in the region $0 \leqslant \operatorname{Arg} z \leqslant \theta$.

Since $|g(x)| \leqslant C \min \left\{1,|x|^{-\delta|x|}\right\}$ in this sector, we may deform the contour of integration, and eventually find that

$$
I_{4}=e^{i \varphi} \int_{0}^{\infty} g(x) \int_{0}^{\infty} e^{-s^{\alpha} t} \frac{e^{i s x e^{-i \theta}}-e^{-s x}}{s} d s d x,
$$

which is identical to the definition of $I_{4}$, except that the integrals are in reverse order.

A very similar argument shows that the order of integration can be reversed in the definition of $I_{5}$, and thus also in $I_{3}$. We conclude that

$$
\int_{0}^{\infty} e^{-s^{\alpha} t} \int_{\mathbb{R}} \frac{F^{-}(s x)}{s} g(x) d x d s=I_{1}+I_{2}+I_{3}=\int_{\mathbb{R}} g(x) \int_{0}^{\infty} e^{-s^{\alpha} t} \frac{F^{-}(s x)}{s} d s d x,
$$

By (5.10), for almost every $t>0$ and every $g \in \mathcal{H}$,

$$
\int_{\mathbb{R}} g(x) \mathbb{P}^{x}\left(\tau_{0}>t\right) d x=\frac{\alpha \sin \frac{\pi}{\alpha}}{\pi \cos \theta} \int_{\mathbb{R}} g(x) \int_{0}^{\infty} \frac{e^{-s^{\alpha} t}}{s} F^{-}(s x) d s d x .
$$

By Lemma 2.5, we have

$$
\mathbb{P}^{x}\left(\tau_{0}>t\right)=\frac{1}{\pi \cos \theta} \int_{0}^{\infty} \frac{e^{-s^{\alpha} t}}{s} F^{-}(s x) d s
$$

for almost all $x \in \mathbb{R} \backslash\{0\}$ and $t>0$. Since both sides are jointly continuous functions of $x \in \mathbb{R} \backslash\{0\}$ and $t>0$ (the right-hand side by a simple application of Lebesgue's dominated convergence theorem), the above equality in fact holds for all $x \in \mathbb{R} \backslash\{0\}$ and $t>0$, and the proof is complete.

Acknowledgments. I express my gratitude to Professor Mateusz Kwaśnicki for his guidance and numerous comments to the preliminary version of this article. 
Spectral theory for stable processes

\section{References}

[1] R. F. Bass, Probabilistic Techniques in Analysis, Springer-Verlag, 1995. MR-1329542

[2] J. Bertoin, Lévy processes, Cambridge University Press, Melbourne-New York, 1996. MR1406564

[3] R. M. Blumenthal, R. K. Getoor, D. B. Ray, On the distribution of first hits for the symmetric stable processes, Trans. Amer. Math. Soc. 99 (1961): 540-554. MR-0126885

[4] K. Bogdan, T. Byczkowski, T. Kulczycki, M. Ryznar, R. Song, Z. Vondraček, Potential Analysis of Stable Processes and its Extensions. Lecture Notes in Mathematics 1980, Springer-Verlag, Berlin-Heidelberg (2009). MR-2569321

[5] A. Borodin, P. Salminen, Handbook of Brownian motion - facts and formulae. Birkhauser, Basel 1996. MR-1477407

[6] L. de Branges, Hilbert spaces of entire functions, Prentice-Hall Series in Modern Analysis, Prentice-Hall, Englewood Cliffs, NJ 1968. MR-0229011

[7] F. Cardero, On the excursion theory for the symmetric stable Lévy processes with index $\alpha \in[1,2]$ and some applications. PhD thesis, Université Pierre et Marie Curie - Paris VI, 2010.

[8] Z.-Q. Chen, P. Kim, T. Kumagai, On heat kernel estimates and parabolic Harnack inequality for jump processes on metric measure spaces, Acta Mathematica Sinica 25, 1067-1089, 2009. MR-2524930

[9] K. L. Chung, Z. Zhao, From Brownian Motion to Schrödinger's Equation, Springer-Verlag, 1995. MR-1329992

[10] R. A. Doney, Hitting probabilities for spectrally positive Lévy processes, J. London Math. Soc., 44, No. 3, 566-576 (1991). MR-1149016

[11] P. L. Duren, Theory of $H^{p}$ Spaces, Academic Press, New York, 1970. MR-0268655

[12] R. K. Getoor, Markov operators and their associated semi-groups, Pacific J. Math. 9(2) (1959): 449-472. MR-0107297

[13] P. Graczyk, T. Jakubowski, On Wiener-Hopf factors for stable processes, Ann. Inst. H. Poincaré Probab. Statist. 47(1) (2011) 9-19. MR-2779394

[14] T. Grzywny, M. Ryznar, Hitting Times of Points and Intervals for Symmetric Lévy Processes, Potential Anal. 46 (2017): 739-777. MR-3636597

[15] S. Havinson, Analytic functions of bounded type, Math. Analysis 1963, pp. 5-80, Akad. Nauk SSSR, Inst. Naucn. Informacii, Moscow, 1965 (in Russian). MR-0196096

[16] T. Juszczyszyn, M. Kwaśnicki, Hitting times of points for symmetric Lévy processes with completely monotone jumps, Electron. J. Probab. 20 (2015), paper no. 48, 24 pp. doi:10.1214/EJP.v20-3440. MR-3339868

[17] H. Kesten, A convolution equation and hitting probabilities of single points for processes with stationary independent increments. Bull. Amer. Math. Soc. 75(3) (1969) 573-578. MR0251797

[18] T. Kulczycki, M. Kwaśnicki, J. Małecki, A. Stos, Spectral properties of the Cauchy process on half-line and interval, Studia Math. 206(3) (2011): 211-271.

[19] A. Kuznetsov, On extrema of stable processes, Ann. Probab. 39(3) (2011) 1027-1060. MR2789582

[20] A. Kuznetsov, A. E. Kyprianou, J. C. Pardo, A. R. Watson, The hitting time of zero for a stable process. Electron. J. Probab. 19 (2014), paper no. 30, 26 pp. doi:10.1214/EJP.v19-2647. MR-3183574

[21] A. Kuznetsov, M. Kwaśnicki, Spectral analysis of stable processes on the positive half-line, Electron. J. Probab. 23 (2018), paper no. 10, 29 pp. doi:10.1214/18-EJP134. MR-3771747

[22] M. Kwaśnicki, Spectral analysis of subordinate Brownian motions on the half-line, Studia Math. 206(3) (2011): 211-271. MR-2860308

[23] M. Kwaśnicki, Spectral theory for symmetric one-dimensional Lévy processes killed upon hitting the origin, Electron. J. Probability 17 (2012), no. 83, 1-29. MR-2988398 
Spectral theory for stable processes

[24] M. Kwaśnicki, Rogers functions and fluctuation theory, (2013), unpublished, arXiv:1312.1866v1.

[25] M. Kwaśnicki, Fluctuation theory for Lévy processes with completely monotone jumps, Electron. J. Probab. 24 (2019), no. 40. MR-3940770

[26] M. Kwaśnicki, J. Małecki, M. Ryznar, First passage times for subordinate Brownian motions, Stoch. Proc. Appl. 123 (2013): 1820-1850. MR-3027903

[27] A. E. Kyprianou, J. C. Pardo, A. R. Watson, Hitting distributions of $\alpha$-stable processes via path censoring and self-similarity, Ann. Probab. 42 (2014), no. 1, 398-430. doi:10.1214/12-AOP790. MR-3161489

[28] J. Letemplier, T. Simon, Unimodality of Hitting Times for Stable Processes. Lecture Notes in Mathematics. 2123. Séminaire de Probabilités XLVI 10.1007/978-3-319-11970-0-13, 2013, 345-359. MR-3330824

[29] P. Mandl, Analytical treatment of one-dimensional Markov processes, Springer-Verlag, Prague, 1968. MR-0247667

[30] M. Marcus, J. Rosen, Markov Processes, Gaussian Processes, and Local Times. Cambridge Univ. Press, New York, 2006. MR-2250510

[31] Y. Ogura, Spectral repesentation for branching processes on the real half-line. Publ. Res. Inst. Math. Sci., 5, 1969: 423-441. MR-0258138

[32] Anthony G. Pakes, A hitting time for Lévy processes, with application to dams and branching processes, Annales de la Faculté des sciences de Toulouse : Mathématiques, Serie 6, Volume 5(3) (1996): 521-544. MR-1440948

[33] P. Patie, M. Savov, Spectral expansions of non-self-adjoint generalized Laguerre semigroups. Mem. Amer. Math. Soc., 2019, 179.

[34] G. Peskir, The Law of the Hitting Times to Points by a Stable Lévy Process with No Negative Jumps. Electron. Commun. Probab. 13 (2008), paper no. 60, 653-659. doi:10.1214/ECP.v131431. MR-2466193

[35] S. C. Port, Hitting times and potentials for recurrent stable processes. J. Anal. Math. 20(1) (1967): 371-395. MR-0217877

[36] C. Profeta, T. Simon, On the Harmonic Measure of Stable Processes. In: C. Donati-Martin , A. Lejay, A. Rouault (eds), Séminaire de Probabilités XLVIII. Lecture Notes in Mathematics, vol 2168. Springer, Cham, 2016. MR-3618135

[37] L.C.G. Rogers, Wiener-Hopf factorization of diffusions and Lévy processes., Proc. London Math. Soc. 47(3) (1983): 177-191. MR-0698932

[38] B.A. Rogozin, The distribution of the first hit for stable and asymptotically stable walks on an interval. Theory Probab. Appl. 17 (1972): 332-338. MR-0300349

[39] M. Rosenblum, J. Rovnyak, Topics in Hardy Classes and Univalent Functions, Springer Basel AG, 1994. MR-1307384

[40] U. Rosler, Unimodality of Passage Times for One-Dimensional Strong Markov Processes. Ann. Probab. 8 (1980), no. 4, 853-859. doi:10.1214/aop/1176994672.

[41] Ken-Iti Sato, Lévy Processes and Infinitely Divisible Distributions, Cambridge University Press, Cambridge, 1999. MR-1739520

[42] R. L. Schilling, R. Song, Z. Vondraček, Bernstein functions. Theory and applications. 2nd Edition, De Gruyter, Göttingen, 2012. MR-2978140

[43] T. Simon, Hitting densities for spectrally positive stable processes, Stochastics An International Journal of Probability and Stochastic Processes, 83:02, 2011: 203-214. MR-2800088

[44] V. S. Vladimirov, Methods of the theory of generalized functions. Taylor and Francis, New York, 2002. MR-2012831

[45] Z. Vondraček, Basic potential theory of certain nonsymmetric strictly alpha-stable processes, Glasnik matematički 37, 1 (2002): 211-233. MR-1918106

[46] K. Yano, Y. Yano, M. Yor, On the Laws of First Hitting Times of Points for One-Dimensional Symmetric Lévy Processes without Gaussian Part. Séminaire de Probabilités XLII, Lecture Notes in Math. 1979, Springer, 2009. MR-2599211 
Spectral theory for stable processes

[47] T. Zhang, X. Zhou, Stochastic analysis and applications to finance : essays in honour of Jia-an Yan. World Scientific, 2012. MR-2976662 


\section{Electronic Journal of Probability Electronic Communications in Probability}

\section{Advantages of publishing in EJP-ECP}

- Very high standards

- Free for authors, free for readers

- Quick publication (no backlog)

- Secure publication $\left(\mathrm{LOCKSS}^{1}\right)$

- Easy interface (EJMS²)

\section{Economical model of EJP-ECP}

- Non profit, sponsored by $\mathrm{IMS}^{3}, \mathrm{BS}^{4}$, ProjectEuclid ${ }^{5}$

- Purely electronic

\section{Help keep the journal free and vigorous}

- Donate to the IMS open access fund ${ }^{6}$ (click here to donate!)

- Submit your best articles to EJP-ECP

- Choose EJP-ECP over for-profit journals

\footnotetext{
${ }^{1}$ LOCKSS: Lots of Copies Keep Stuff Safe http://www. lockss.org/

${ }^{2}$ EJMS: Electronic Journal Management System http://www.vtex.lt/en/ejms.html

${ }^{3}$ IMS: Institute of Mathematical Statistics http://www.imstat.org/

${ }^{4}$ BS: Bernoulli Society http://www. bernoulli-society.org/

${ }^{5}$ Project Euclid: https://projecteuclid.org/

${ }^{6}$ IMS Open Access Fund: http://www.imstat.org/publications/open.htm
} 